ilectromagnetic Wave Interactions, pp. 1-37

'clited by A. Guran, R. Mittra and P. J. Moser

íeries on Stability, Vibration and Control of Systems Series B: Vol. 12

(c) World Scientific Publishing Company

\title{
CLOSED-FORM GREEN'S FUNCTIONS AND THEIR USE IN THE METHOD OF MOMENTS
}

\author{
M. I. AKSUN \\ Electrical and Electronics Eng. Dept. \\ Bilkent University \\ Ankara 06533, Turkey \\ and \\ R. MITTRA \\ Electromagnetic Communication Laboratory \\ Electrical and Computer Eng. Dept. \\ University of Illinois at Urbana-Champaign \\ 1406 W. Green Street \\ Urbana, IL 61801
}

\begin{abstract}
Derivation of the spatial-domain, closed-form Green's functions of the vector and scalar potentials are demonstrated for planar media, and their use in conjunction with the method of moments (MoM) is presented. As the first step of the derivation, the Green's functions are obtained analytically in the spectral domain for various sources viz., horizontal and vertical electric and magnetic dipoles embedded in a planar stratified media. The spatial-domain Green's function can be obtained from the Sommerfeld integral which is the Hankel transform of the corresponding Green's function in the spectral domain. The analytical evaluation of this transformation yields the closed-form, spatial-domain Green's functions which can be used in the solution of a mixed-potential integral equation (MPIE) via the MoM. This combination, i.e., the use of the closed-form Green's functions in conjunction with the MoM, results in a significant improvement in the fill-time of MoMl matrices. In the conventional application of the spatial-domain $M \circ M$, the matrix elements are double integrals and they require the evaluation of the time-consuming Sommerfeld integral for the spatial-domain Green's function. In the approach presented herein, the spatial-domain Green's functions are in closed forms, and the remaining double-integrals in the matrix elements are evaluated analytically. Thus, there are two factors in this approach that contribute to the improvement in the computation time: (i) elimination of the numerical integration to obtain the spatial-domain Green's functions; (ii) circumventing the need to carry out the numerical integration in the calculation of the MoM matrix elements.
\end{abstract}

\section{Introduction}

Electromagnetic modeling of printed structures in a stratified medium is an important problem in computational electromagnetics, and has recently attracted widespread attention. This is attributable to the increased use of multilayer microstrip geometries in various applications of microstrip antennas ${ }^{1-6}$, and in monolithic microwave integrated circuits ${ }^{i, 8}$. Therefore, a considerable amount of interest has been focused 
on the development of a rigorous and yet computationally efficient computer-aided design tools for microstrip geometries in layered media. Hence, a variety of numerical techniques for accurate modeling and simulation of the electrical performances of such circuits have been proposed and studied extensively. These include the method of moments $(\mathrm{MoM})^{9}$ and its variants; the finite element method (FEM) ${ }^{10}$; the finite difference time domain (FDTD) method ${ }^{11}$, and quasi-static methods based on conformal mapping ${ }^{12}$. Among these approaches, the spatial and spectral domain MoM approaches are the most commonly used numerical techniques for the rigorous analysis of printed geometries in multilayer planar media.

The use of the MoM in the analysis of microstrip geometries requires the computation of the Green's functions for layered media, which are represented analytically in the spectral domain and transformed into the spatial domain from the spectraldomain representations via the Hankel transform. The resulting spatial-domain representation, also referred to as the Sommerfeld integral ${ }^{13}$, is a one-dimensional integral over an infinite range with the Hankel function as its kernel; hence, its numerical evaluation is computationally inefficient unless an acceleration technique is used. Since the Green's functions in the spectral domain are expressible in closed forms, the use of the spectral-domain MoM in the analysis of microstrip geometries had attracted much interest ${ }^{14-17}$. Although, relatively speaking, the spectral-domain MoM is more efficient in terms of computation time than some of the other numerically rigorous techniques, it is still quite time-consuming to implement. This is because, in this approach, the MoM matrix elements are double integrals of complex, slowly-decaying functions over infinite ranges. To overcome the difficulty introduced by the slowlydecaying nature of the integrand, the spectral-domain approach is usually employed in conjunction with an acceleration technique in which the asymptotic part of the integrand is subtracted from the original integrand and its contribution is calculated either analytically or in a numerically efficient manner ${ }^{18,19}$. However, even with the use of this tactic, this computation remains expensive, because the integrands are oscillatory functions of the spectral domain variables, and for the self-terms, for which the observation region coincides with the source region, the convergence is still quite slow. The computational inefficiency plagues the spatial domain MoM as well, since it involves the evaluation of at least a three-dimensional integral for the matrix element, one of which is the Sommerfeld integral. However, since the remaining double-integral is over a finite domain, the spatial-domain MoM can be made computationally inexpensive if the Sommerfeld integrals can be evaluated efficiently.

A number of different techniques have been proposed for the efficient evaluation of the Sommerfeld-type integrals by employing acceleration techniques. These include the Shank's transformation, the Euler transformation and the method of averages. Among these, the method of averages has been demonstrated to be the most efficient for the evaluation of the Sommerfeld-type integrals ${ }^{20}$. These techniques require the numerical evaluation of the integral at several intervals that leads to a series, whose convergence is accelerated via a nonlinear transformation. In this approach, it is necessary to perform a numerical integration over a portion of the infinite range of the original integral. Thus the efficiency of this technique is determined by the extent 
of the interval over which one needs to perform the numerical integration to predict the end result sufficiently accurately. In addition, since the Sommerfeld integral is a parametric integral, with the radial distance $\rho$ as the parameter, the process of acceleration described above has to be performed for each value of the parameter, which is usually on the order of hundreds for typical applications of the spatialdomain MoM. Consequently, even if the method of averages enabled us to compute the Sommerfeld integral efficiently for one value of $\rho$, it would be quite expensive to employ this technique directly in conjunction with the spatial-domain MoM which calls the evaluation of the above integral for many $\rho$. One approach to circumventing this difficulty is to precompute the values of the Green's functions for a range of radial distances and curve-fit these values locally using low-order polynomials ${ }^{21}$. Even so, the integral must be evaluated for tens of radial distances for the purpose of curvefitting, because of the sharp changes in some Green's functions caused by phase cancelations.

Recently, yet another approach has been proposed which appears to resolve all of these difficulties mentioned above. This approach was first proposed ${ }^{22}$ for a thick, single layer microstrip geometry, and later extended to multilayer planar geometries with arbitrary thicknesses ${ }^{23}$, 24 . It takes advantage of the fact that the Sommerfeld integral can be integrated analytically if the integrand, apart from the kernel $H_{\circ}^{(2)}\left(k_{\rho} \rho\right)$, can be approximated by complex exponentials. With this approach, the numerical integration, which causes computational inefficiency in the acceleration techniques mentioned above, is completely eliminated. Instead, the burden of the numerical algorithm falls on the task of approximating the spectral-domain Green's functions in terms of complex exponentials. Naturally, one might raise the questions regarding the computational efficiency and robustness of this technique and the difficulties arising in the process of applying the algorithms for the exponential approximation. Before addressing these questions, it would be useful to describe some of the recent improvements ${ }^{25}$ introduced in the technique. In its original form ${ }^{22}$ Prony's method was used for the exponential approximation ${ }^{26}$, which required the same number of samples as the number of unknowns, i.e., twice the number of complex exponentials (one for the coefficient and one for the exponent). One consequence of this was that it was difficult to account for the rapid variations of the integrand in the spectral domain without using a large number of complex exponentials, and this phenomenon is attributable, in part, to the uniform sampling required in Prony's method. Although the least-square Prony's method improves its ability to account for the rapid changes with a moderate number of exponentials ${ }^{23}$, it still requires several trial and error iterations, because of the noise sensitivity of the Prony methods ${ }^{27}$, which render the technique to be inefficient and not robust. As a solution, another exponential approximation technique, called the generalized pencil of function (GPOF) $\operatorname{method}^{28}$, is employed in casting the Green's functions into closed forms ${ }^{24}$. The GPOF method has turned out to be quite robust and less noise sensitive when compared to the original and least-square Prony methods, and also provides a good measure for choosing the number of exponentials used in the approximation. However, it still requires one to study in advance the spectral-domain behavior of the Green's function in order 
to decide on the approximation parameters like the number of sampling points and the maximum value of the sampling range. In addition, since the approximation techniques, such as the Prony and the GPOF methods, require the function to be sampled uniformly, one would need to take thousands of samples in order to be able to approximate a slow converging function with rapid changes (even if this were to occur in a small region), which is a typical behavior of the spectral-domain Green's functions of the scalar potentials in a thin substrate. Because of these difficulties, the technique of deriving the closed-form Green`s functions and subsequently using them in MoM applications is not considered to be robust, and thus could not be used conveniently for the development of a general-purpose electromagnetic software. Recently, a new approach based on a two-level approximation has been proposed to overcome these difficulties, and it has been demonstrated that the new approach is very robust and computationally much more efficient than the original one or its variants. The two-level approach divides the range of approximation into two parts, the first of which covers the region where the function to be approximated has rapid transitions, whereas the function is smooth in the second region. Thus, it is no longer necessary to take thousands of samples to account for a rapid transition that occurs in a small part of the entire range, and this results in a significant reduction of the number of data points to be processed in deriving the exponential approximation, which, in turn, translates into a substantial saving in the computation time. Throughout this chapter, this new approach is referred to as the two-level approach while the original technique is called the one-level approach.

With the introduction of the robust and efficient approach in the derivation of the closed-form Green's functions, the use of the MoM in the spatial domain becomes very attractive for the analysis of printed geometries in a multilayer environment. The Sommerfeld integral is now completely eliminated and the calculation of the matrix elements in the spatial-domain MoM now only requires double integrals over finite ranges that are evaluated numerically. It has been demonstrated that even the elimination of only the Sommerfeld integrals improves the computational efficiency of the spatial-domain MoM significantly ${ }^{29}$. It has also been recently demonstrated ${ }^{30}$ that, with the analytical approximation of the remaining double-integrals, the computational efficiency of the evaluation of the matrix elements is further improved. Consequently, a substantial improvement in the matrix-fill time is achieved by analytically evaluating all of the integrals involved in the spatial-domain Moiv.

Section 2 describes the derivation of the closed-form Green's functions and includes the derivation of the spectral-domain Green's functions, discussions on the difficulties associated with the original one-level approach, and assessment of the robustness and computational efficiency of the two-level approach. Section 3 describes the use of the closed-form Green's functions in conjunction with the MoM and provides the analytical evaluation of the MoM matrix elements together with the necessary integral identities. 


\section{Closed-Form Green's Functions}

Let us consider, for the sake of illustration, a planar, multilayercd medium shown in Fig. 1 where it is assumed that the layers extend to infinity in the transverse directions. The source, (HED, HMD, VED or VMD) is embedded in region $i$ and the observation point can be located in an arbitrary layer. Each layer can have different electric and magnetic properties $\left(\epsilon_{\tau}, \mu_{\tau}\right)$ and thickness $\left(d_{i}\right)$. The perfect electric or magnetic conducting planes and half-space are also regarded as layers in this formulation. The procedure for deriving closed-form expressions for the Green's

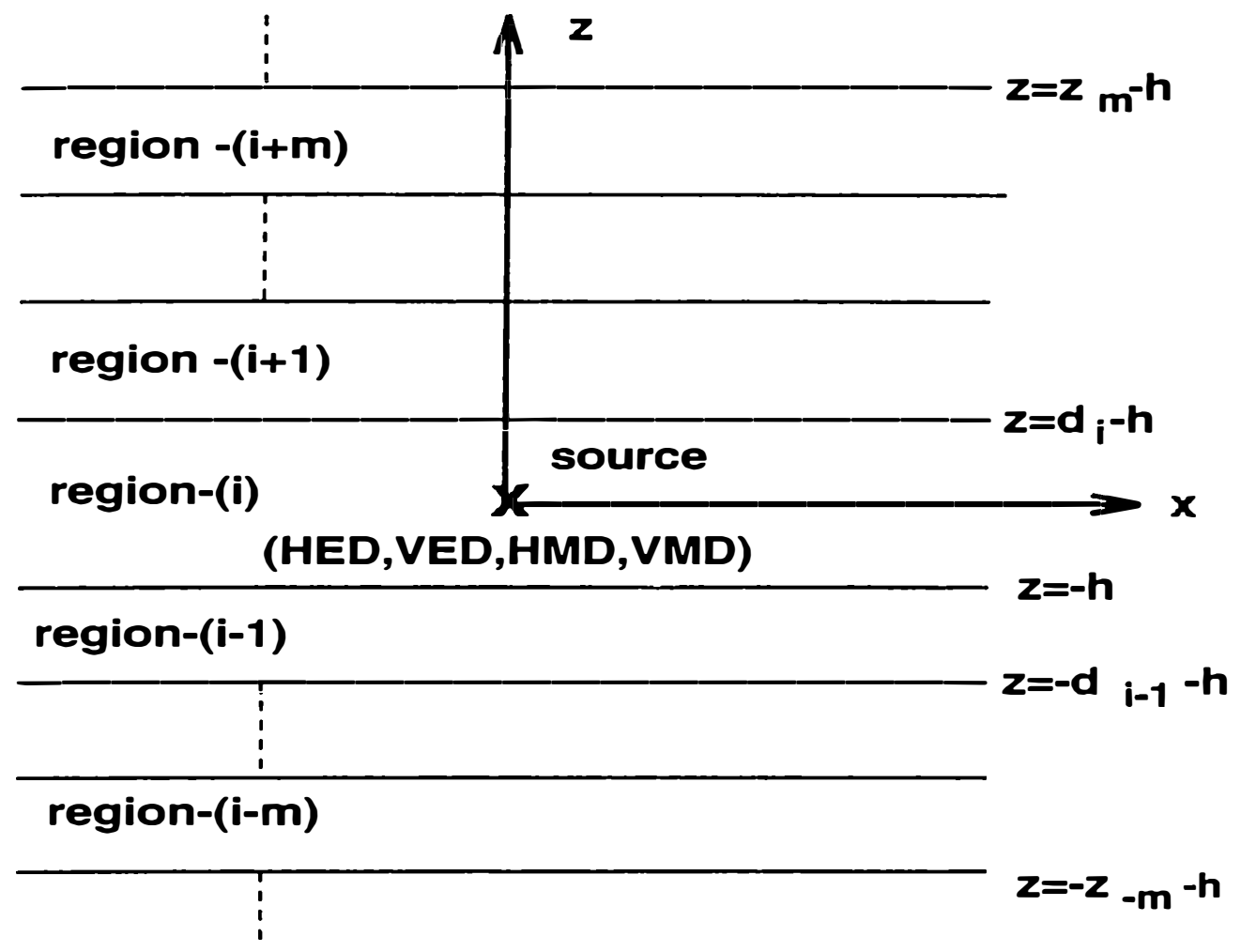

Figure 1: Sources embedded in a multilayer medium.

functions entails the following steps:

1. Derivation of the Green's functions in the spectral domain.

(a) Green's functions are derived in the source layer.

(b) Green's functions in the observation layer are obtained by using an iterative algorithm applied to each TE and TM component of the Green's functions in the source layer.

2. Derivation of the spatial domain, closed-form Green's functions. 
(a) Spectral-domain Green's functions are approximated in terms of complex exponentials obtained from the GPOF method after the direct terms have been extracted.

(b) Closed-form Green's functions are obtained analytically by using the Sommerfeld identity for each of the complex exponentials.

All of the Green's functions, presented herein, are for the vector and scalar potentials that are indeed not defined uniquely in stratified media ${ }^{31,32}$. Therefore, different sets of Green's functions for the vector and scalar potentials can be chosen to satisfy the same boundary conditions. The following notation for the Green's function is commonly used and referred to as the traditional form ${ }^{33}$ :

$$
\overrightarrow{\bar{G}}_{A, F}=(\hat{x} \hat{x}+\hat{y} \hat{y}) G_{x x}+\hat{z} \hat{x} G_{z x}+\hat{z} \hat{y} G_{z y}+\hat{z} \hat{z} G_{z z}
$$

for the vector potentials, and $G_{x, y}^{q_{e, m}}$ and $G_{z}^{q_{e, m}}$ for the scalar potentials. Note that, in this representation, the scalar potentials of the point charges associated with the horizontal and vertical dipoles are not identical. This leads to some difficulties in the solution of the mixed potential integral equation for a geometry where both the horizontal and vertical sources (HED and VED or HMD and VMD) are present at the same point, as in the case of a microstrip etch fed by a vertical probe. To overcome this difficulty, an alternative form has been suggested ${ }^{32}$ for the Green's functions and these representations are given in Appendix A.

\subsection{Green's Functions in the Spectral Domain}

The spectral-domain Green's functions of the vector and scalar potentials can be obtained from the electric and magnetic fields generated by a current dipole $\mathbf{J}=$ $I_{\mathrm{o}} l \delta(\mathbf{r}) \alpha$, where $\alpha$ is a unit vector. For the sake of illustration, the field components for an HED in a multilayer media, Fig. 1, can be written in the source layer (layer $i$ ) as follows ${ }^{34}$ :

$$
\begin{aligned}
& \epsilon_{i} E_{z_{i}}=\frac{I_{0} l}{8 \pi^{2} \omega} \int_{-\infty}^{\infty} \int d k_{x} d k_{y} k_{x} e^{-j \mathbf{k}_{\rho} \cdot\left(\rho-\rho^{\prime}\right)}\left[ \pm e^{-j k_{z_{i}}|z|}+B_{h}^{e} e^{j k_{z_{i}} z}+D_{h}^{e} e^{-j k_{z_{i}} z}\right] \\
& \mu_{i} H_{z_{i}}=\mu_{i} \frac{I_{0} l}{8 \pi^{2}} \int_{-\infty}^{\infty} \int d k_{x} d k_{y} \frac{k_{y}}{k_{z_{i}}} e^{-j \mathbf{k}_{\rho} \cdot\left(\rho-\rho^{\prime}\right)}\left[e^{-j k_{z_{\mathrm{i}}}|z|}+A_{h}^{e} e^{j k_{z_{i}} z}+C_{h}^{e} e^{-j k_{z_{i}} z}\right]
\end{aligned}
$$

where the $z$ dependence of the fields in the source region is written as the sum of the direct term and up- and down-going waves due to the reflections from the boundaries at $z=-h$ and $z=d_{i}-h$, respectively, and + and - signs are for $z>0$ and $z<0$, respectively. The coefficients of the up- and down-going waves can be obtained in terms of the generalized reflection coefficients by applying the appropriate boundary conditions: (i) the down-going waves for $z>0$ are the consequence of the reflections of the up-going waves at $z=d_{i}-h$; and (ii) the up-going waves for $z<0$ are the 
consequence of the reflections of the down-going waves at $z=-h$. It should be noted that the other field components can be easily derived from the z-components of the fields ${ }^{34}$, so they are not included here. After having obtained the field components, the components of the vector potentials and the scalar potential can be derived from the following relations:

$$
\begin{array}{r}
\nabla \times \mathbf{A}=\mu_{i} \mathbf{H} \\
\phi_{d}=-\frac{\nabla \times \mathbf{A}}{j \omega \mu_{i} \epsilon_{i}}=\frac{I_{0} l \quad \partial \phi}{j \omega \partial l^{\prime}}
\end{array}
$$

where $\phi_{d}$ and $\phi$ are the scalar potentials for the dipole element and a point charge, respectively, and $l^{\prime}$ is replaced by $x^{\prime}$ or $y^{\prime}$ for an HED (depending upon the orientation of the dipole) and replaced by $z^{\prime}$ for a VED. Given below are the expressions of the spectral-domain Green's functions (traditional form) in the source layer for HED, HMD, VED and VMD sources. They read:

HED:

$$
\begin{aligned}
& \tilde{G}_{x x}^{A}=\frac{\mu_{i}}{2 j k_{z_{i}}}\left[e^{-j k_{z_{i}}|z|}+A_{h}^{e} e^{j k_{z_{\mathrm{i}}} z}+C_{h}^{e} e^{-j k_{z_{i}} z}\right]
\end{aligned}
$$

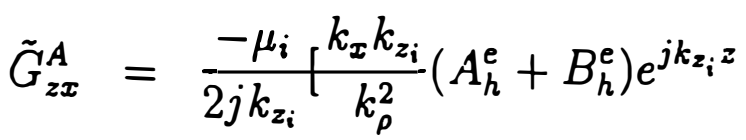

$$
\begin{aligned}
& \left.+\frac{k_{x} k_{z_{i}}}{k_{\rho}^{2}}\left(D_{h}^{e}-C_{h}^{e}\right) e^{-j k_{z_{i}} z}\right] \\
& \tilde{G}_{x}^{q_{e}}=\frac{1}{j 2 \epsilon_{i} k_{z_{i}}}\left[e^{-j k_{z_{i}}|z|}+\frac{k_{z_{i}}^{2} B_{h}^{e}+k_{i}^{2} A_{h}^{e}}{k_{\rho}^{2}} e^{j k_{z_{\mathrm{i}} z}}\right. \\
& \left.+\frac{k_{i}^{2} C_{h}^{e}-k_{z_{i}}^{2} D_{h}^{e}}{k_{\rho}^{2}} e^{-j k_{z_{\mathrm{i}}} z}\right]
\end{aligned}
$$

HMD:

$$
\begin{aligned}
& \tilde{G}_{x x}^{F}=\frac{\epsilon_{i}}{2 j k_{z_{i}}}\left[e^{-j k_{z_{i}}|z|}+A_{h}^{m} e^{j k_{z_{i}} z}+C_{h}^{m} e^{-j k_{z_{i}} z}\right]
\end{aligned}
$$

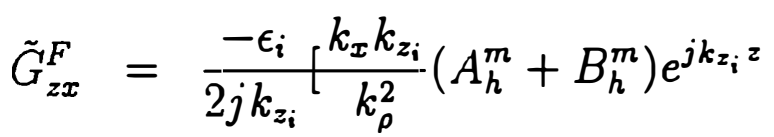

$$
\begin{aligned}
& \left.+\frac{k_{x} k_{z_{i}}}{k_{\rho}^{2}}\left(D_{h}^{m}-C_{h}^{m}\right) e^{-j k_{z_{i} z} z}\right] \\
& \tilde{G}_{x}^{q_{m}}=\frac{1}{j 2 \mu_{i} k_{z_{i}}}\left[e^{-j k_{z_{i}}|z|}+\frac{k_{z_{i}}^{2} B_{h}^{m}+k_{i}^{2} A_{h}^{m}}{k_{\rho}^{2}} e^{j k_{z_{i}} z}\right. \\
& \left.+\frac{k_{i}^{2} C_{h}^{m}-k_{z_{i}}^{2} D_{h}^{m}}{k_{\rho}^{2}} e^{-j k_{z_{i}} z}\right]
\end{aligned}
$$


VED:

$$
\begin{aligned}
& \tilde{G}_{z z}^{A}=\frac{\mu_{i}}{j 2 k_{z_{i}}}\left[e^{-j k_{z_{\mathrm{i}}}|z|}+A_{v}^{e} e^{-j k_{z_{\mathrm{i}} z} z}+B_{v}^{e} e^{j k_{z_{\mathrm{i}}} z}\right] \\
& \tilde{G}_{z}^{q_{e}}=\frac{1}{j 2 k_{z_{\mathrm{i}}} \epsilon_{i}}\left[e^{-j k_{z_{\mathrm{i}}}|z|}+C_{v}^{e} e^{-j k_{z_{\mathrm{i}} z} z}+D_{v}^{e} e^{j k_{z_{\mathrm{i}} z} z}\right]
\end{aligned}
$$

VMD:

$$
\begin{gathered}
\tilde{G}_{z z}^{F}=\frac{\epsilon_{i}}{j 2 k_{z_{i}}}\left[e^{-j k_{z_{i}}|z|}+A_{v}^{m} e^{-j k_{z_{i}} z}+B_{v}^{m} e^{j k_{z_{i}} z}\right] \\
\tilde{G}_{z}^{q_{m}}=\frac{1}{j 2 k_{z_{i}} \mu_{i}}\left[e^{-j k_{z_{i}}|z|}+C_{v}^{m} e^{-j k_{z_{i}} z}+D_{v}^{m} e^{j k_{z_{i}} z}\right]
\end{gathered}
$$

where $\tilde{G}_{i j}^{A, F}$ denotes the spectral-domain Green's functions for the vector potentials in the direction- $i$ due to a unit $j$-directed current element; $\tilde{G}_{i}^{q_{e, m}}$ represents the Green's function of the scalar potential in the spectral domain due to a unit $i$-directed electric or magnetic current element; $k_{i}^{2}=k_{\rho}^{2}+k_{z_{i}}^{2}$; the superscripts $A$ and $F$ represent the magnetic and the electric vector potentials, respectively; and, $q_{e}$ and $q_{m}$ represent the electric and magnetic scalar potentials, respectively. The coefficients, $A_{h, v}^{e, m}, B_{h, v}^{e, m}$, $C_{h, v}^{e, m}, D_{h, v}^{e, m}$ are functions of the generalized reflection coefficients $\tilde{R}_{T E, T M}$, and are given by

$$
\begin{aligned}
A_{h}^{e, m} & =e^{-j k_{z_{i}}\left(d_{i}-h\right)} \tilde{R}_{T E, T M}^{i, i+1}\left[e^{-j k_{z_{i}}\left(d_{i}-h\right)}\right. \\
& \left.+\tilde{R}_{T E, T M}^{i, i-1} e^{-j k_{z_{i}}\left(d_{i}+h\right)}\right] M_{i}^{T E, T M} \\
B_{h}^{e, m} & =e^{-j k_{z_{i}}\left(d_{i}-h\right)} \tilde{R}_{T M, T E}^{i, i+1}\left[e^{-j k_{z_{i}}\left(d_{i}-h\right)}\right. \\
& \left.-\tilde{R}_{T M, T E}^{i, i-1} e^{-j k_{z_{i}}\left(d_{i}+h\right)}\right] M_{i}^{T M, T E} \\
C_{h}^{e, m} & =e^{-j k_{z_{i}} h} \tilde{R}_{T E, T M}^{i, i-1}\left[e^{-j k_{z_{i}} h}\right. \\
& \left.+\tilde{R}_{T E, T M}^{i, i+1} e^{-j k_{z_{i}}\left(2 d_{i}-h\right)}\right] M_{i}^{T E, T M} \\
D_{h}^{e, m} & =e^{-j k_{z_{i}} h} \tilde{R}_{T M, T E}^{i, i-1}\left[-e^{-j k_{z_{i}} h}\right. \\
& \left.+\tilde{R}_{T M, T E}^{i, i+1} e^{-j k_{z_{i}}\left(2 d_{i}-h\right)}\right] M_{i}^{T M, T E} \\
A_{v}^{e, m} & =e^{-j k_{z_{i}} h} \tilde{R}_{T M, T E}^{i, i-1}\left[e^{-j k_{z_{i}} h}\right. \\
& \left.+\tilde{R}_{T M, T E}^{i, i+1} e^{-j k_{z_{i}}\left(2 d_{i}-\dot{n}\right)}\right] M_{i}^{T M . T E}
\end{aligned}
$$




$$
\begin{aligned}
B_{v}^{e, m} & =e^{-j k_{z_{i}}\left(d_{i}-h\right)} \tilde{R}_{T M, T E}^{i, i+1}\left[e^{-j k_{z_{i}}\left(d_{i}-h\right)}\right. \\
& \left.+\tilde{R}_{T M, T E}^{i, i-1} e^{-j k_{z_{i}}\left(d_{i}+h\right)}\right] M_{i}^{T M, T E} \\
C_{v}^{e, m} & =e^{-j k_{z_{i}} h} \tilde{R}_{T M, T E}^{i, i-1}\left[-e^{-j k_{z_{i}} h}\right. \\
& \left.+\tilde{R}_{T M, T E}^{i, i+1} e^{-j k_{z_{i}}\left(2 d_{i}-h\right)}\right] M_{i}^{T M, T E} \\
D_{v}^{e, m} & =e^{-j k_{z_{i}}\left(d_{i}-h\right)} \tilde{R}_{T M, T E}^{i, i+1}\left[-e^{-j k_{z_{i}}\left(d_{i}-h\right)}\right. \\
& \left.+\tilde{R}_{T M, T E}^{i, i-1} e^{-j k_{z_{i}}\left(d_{i}+h\right)}\right] M_{i}^{T M, T E}
\end{aligned}
$$

where

$$
\begin{gathered}
M_{i}^{T E, T M}=\left[1-\tilde{R}_{T E, T M}^{i, i+1} \tilde{R}_{T E, T M}^{i, i-1} e^{-j k_{z_{\mathrm{i}}} 2 d_{\mathrm{i}}}\right]^{-1} \\
\tilde{R}_{T E, T M}^{j+1, j}=\frac{R_{T E, T M}^{j+1, j}+\tilde{R}_{T E, T M}^{j, j-1}}{1-R_{j, j+1} \tilde{R}_{T E, T M}^{j, j-1} e^{-j k_{z_{j}} 2 d_{j}}}
\end{gathered}
$$

Here $R$ and $\tilde{R}$ are the Fresnel and generalized reflection coefficients ${ }^{34}$ for which the subscripts $T E$ and $T M$ represent the polarization of the wave, and the superscripts $(i, i-1)$ or $(i, i+1)$ show the layer numbers. The subscripts $h$ and $v$ used in the coefficients (16-23) represent the orientation of the source, horizontal and vertical, respectively, while the superscripts $e$ and $m$ denote the type of the source, electric and magnetic, respectively. It should be noted that the horizontal Green's functions for the $y$-oriented dipoles can be obtained simply by setting $\tilde{G}_{y y}^{A, F}=\tilde{G}_{x x}^{A, F}, \frac{\tilde{G}_{z y}^{A, F}}{k_{y}}=\frac{\bar{G}_{x}^{A, F}}{k_{x}}$, and $\tilde{G}_{y}^{q_{e, m}}=\tilde{G}_{x}^{q_{e, e, m}}$.

The amplitudes of the up- and down-going waves in a layer different from the source layer are related to those in the adjacent layers by,

$$
A_{j}^{-}=A_{j+1}^{-} \frac{T_{j+1, j} e^{-j\left(k_{z_{j+1}}-k_{z_{j}}\right)\left(h+z_{-m+1}\right)}}{1-R_{j, j+1} \tilde{R}_{j, j-1} e^{-j k_{z_{j}} 2 d_{j}}}
$$

where $A_{j}^{-}$and $A_{j+1}^{-}$are the amplitudes of the down-going waves in layers $j$ and $j+1$, respectively, $(j=i-m), T$ is the transmission coefficient, and $z_{-m}$ is the distance between the lower boundary of the source layer $i$ and the lower boundary of layer $j$, Fig. 1. Similarly the amplitudes of the up-going waves in layer $j=i+m$ can be written as

$$
A_{j}^{+}=A_{j-1}^{+} \frac{T_{j-1, j} e^{-j\left(k_{z_{j-1}}-k_{z_{j}}\right)\left(z_{m-1}+d_{i}-h\right)}}{1-R_{j, j-1} \tilde{R}_{j, j+1} e^{-j k_{z_{j}} 2 d_{j}}}
$$

Therefore, starting from the source layer, the field expressions for any layer can be obtained iteratively. 


\subsection{Closed-Form Green's Functions in the Spatial Domain}

Since the principal goal of this section is to introduce a robust and efficient technique to obtain the spatial-domain Green's functions in closed-forms for planar layered media, it would be useful to first provide the definition of the spatial-domain Green's functions

$$
G^{A, F, q_{e}, q_{m}}=\frac{1}{4 \pi} \int_{S I P} d k_{\rho} k_{\rho} H_{o}^{(2)}\left(k_{\rho} \rho\right) \tilde{G}^{A, F, q_{e}, q_{m}}\left(k_{\rho}\right)
$$

where, $G$ and $\tilde{G}$ are the Green's functions in the spatial and spectral domains, respectively, $H_{o}^{(2)}$ is the Hankel function of the second kind and SIP is the Sommerfeld integration path defined in Fig. 2. The Sommerfeld integral given in (28) cannot be

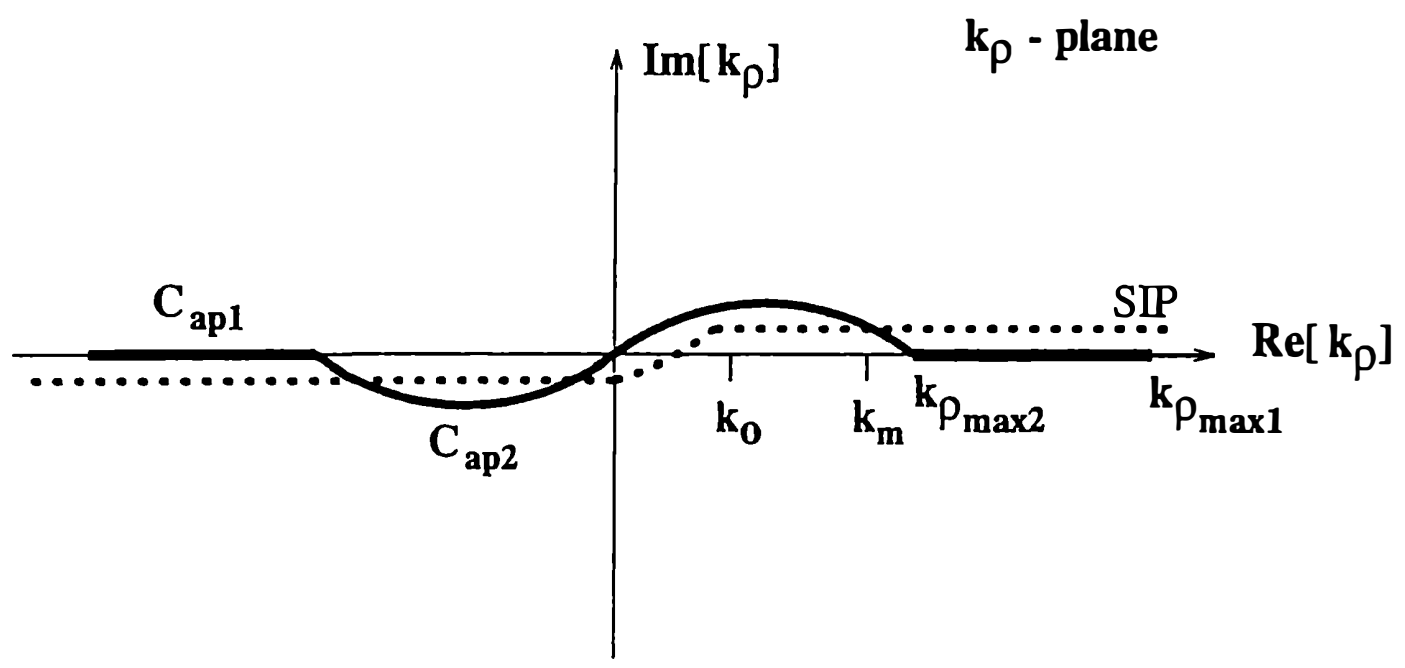

Figure 2: Definition of the Sommerfeld integration path, and the paths $C_{a p 1}$ and $C_{a p 2}$ used in one- and two-level approximations.

integrated analytically, except for a few special cases. However, if $\tilde{G}$, the spectral domain representation of the Green's function in the integrand can be approximated in terms of complex exponentials, the analytical evaluation of the integral (28) becomes possible via the Sommerfeld identity

$$
\frac{e^{-j k r}}{r}=-\frac{j}{2} \int_{S I P} d k_{\rho} k_{\rho} H_{o}^{(2)}\left(k_{\rho} \rho\right) \frac{e^{-j k_{z}|z|}}{k_{z}} .
$$

Therefore, the crucial step in the derivation of the closed-form Green's functions is the exponential approximation of $\tilde{G}$. Since the approximation techniques used for this problem, namely the original Prony, the least square Prony and the GPOF methods, require uniform samples along a real variable of a complex-valued function, one might think of choosing the integration path in (28) along the real $k_{\rho}$ axis so that $\tilde{G}$ can be sampled along a real variable. However, one should note that $k_{z}^{2}=k^{2}-k_{\rho}^{2}$ and sampling along the real $k_{\rho}$ axis results in an approximation in terms of exponentials of $k_{\rho}$ which cannot be cast into a form of exponentials of $k_{z}$ as required in the application 
of the Sommerfeld identity (29). Hence, the SIP must be deformed such that the mapping is from a real variable, viz., the running parameter of the mapping, onto the complex $k_{z}$ plane. In the original approach, a deformed path on $k_{\rho}$ plane, denoted by $C_{a p 2}$ in Fig. 2, was used to sample the function to be approximated, while in the new approach, called the two-level approach, a path formed by the paths $C_{a p 2}$ and $C_{a p 1}$ is employed. The details of these approaches together with the advantages and disadvantages will be discussed in the following sections.

For a general-purpose algorithm, the spectral-domain Green's functions are obtained for a multilayer medium and neither surface wave poles nor the real images are extracted. The extraction of the surface-wave poles (SWP) and the real images helps the exponential approximation techniques by rendering the Green's functions in the spectral domain well-behaved and rapidly converging functions. However, since the contribution of the SWPs is small for geometries on a thin substrate, and it is not possible to find the real images for multilayer planar structures analytically except for some simple cases such as single and double layers, it is likely that the advantage gained by manipulating the Green's functions in a manner described above would be limited to a restricted class of planar geometries and would not lead to a general-purpose or robust algorithm.

Note that to be able to use Sommerfeld identity, the approximation of the spectral domain Green's functions, Eqs. (6)-(15), must be performed for the terms with the square brackets, i.e., the terms other than those that are $\frac{1}{k_{z_{1}}}$. In addition, $k_{x}$ and $k_{y}$ parameters in $\tilde{G}_{z x}^{A, F}$ and $\tilde{G}_{z y}^{A, F}$, respectively, are excluded in the approximation and their contributions are added in the spatial domain (after having obtained the spatial domain representations of $\frac{\bar{G}_{x, F}^{A, F}}{k_{x}}$ and $\frac{\bar{G}_{z y}^{A}, F}{k_{y}}$.) by differentiating them analytically with respect to $x$ and $y$, respectively.

\subsubsection{Original One-Level Approximation}

The deformed path $C_{a p 2}$ on the $k_{\rho}$ plane is defined as a mapping of a real variable $t$ onto the complex $k_{z}$ plane by

$$
k_{z_{i}}=k_{i}\left[-j t+\left(1-\frac{t}{T_{\mathrm{o} 2}}\right)\right], \quad 0 \leq t \leq T_{\mathrm{o} 2}
$$

The Green's functions are sampled uniformly on $t \in\left[0, T_{\text {o2 }}\right]$, which maps onto the path $C_{a p 2}$ with $k_{\rho_{\max 2}}=k_{i}\left[1+T_{\mathrm{o} 2}^{2}\right]^{1 / 2}$ in the $k_{\rho}$-plane, and then approximated in terms of exponentials of $t$ which can be easily transformed into exponentials of $k_{z_{i}}$. This scheme is called the one-level approximation approach because the complex function to be approximated is sampled between 0 and $T_{\text {o2 }}$, and is assumed to be negligible beyond $T_{\text {o2 }}$.

It is instructive to consider the practical details of the implementation of the exponential approximation along the path defined in Eq. (30). It is of utmost importance for the success of this approach to choose, judiciously, the approximation parameters, viz., the interval $T_{02}$, the number of exponentials to be used in the approximation, 
and the number of samples in $t \in\left[0, T_{\mathrm{o} 2}\right]$. To illustrate the implementation of the one-level exponential approximation and the difficulties involved, the spectral-domain Green's function for the scalar potential due to an x-directed dipole, $\tilde{G}_{x}^{q}$, is given in Fig. 3, for a geometry of four layers at $30 \mathrm{GHz}$ : 1st layer- PEC; 2nd layer- $\epsilon_{r 2}=12.5$, $d_{2}=0.03 \mathrm{~cm} ; 3$ rd layer- $\epsilon_{r 3}=2.1, d_{3}=0.07 \mathrm{~cm} ; 4$ th layer- free-space, and the source and observation planes are chosen at the interface of the second and third layers. It is

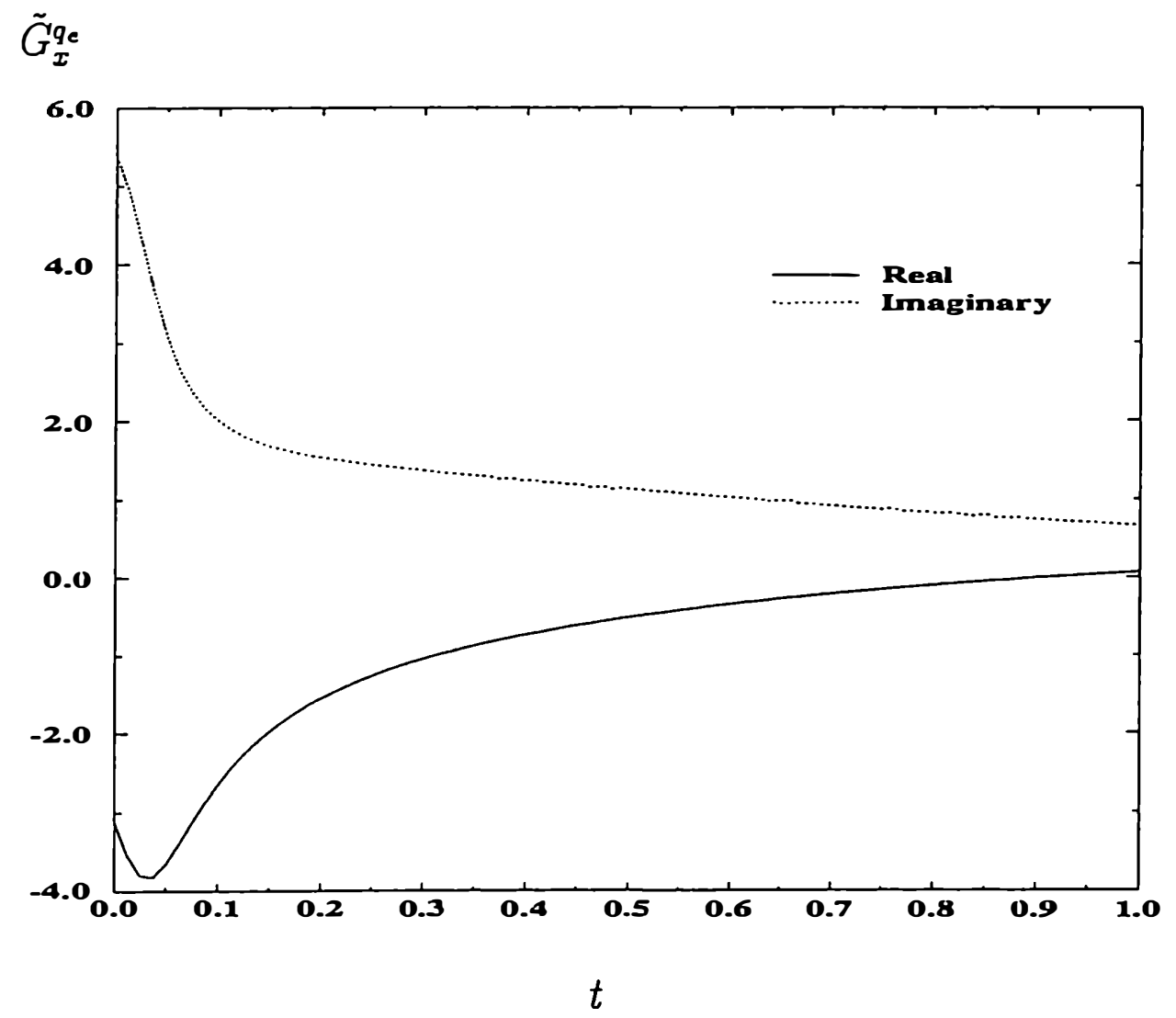

Figure 3: The magnitude of the spectral-domain Green's function $\tilde{G}_{x}^{q_{e}}$ along the path $C_{a p 2}$. 1st layer- PEC; 2 nd layer- $\epsilon_{r 2}=12.5, d_{2}=0.03 \mathrm{~cm} ; 3$ rd layer- $\epsilon_{r 3}=2.1, d_{3}=0.07$ $\mathrm{cm} ; 4$ th layer- free-space, freq $=30 \mathrm{GHz}$.

evident from Fig. 3 that Green's functions can have sharp peaks and fast changes for small $t$, which maps to the far-field region in the spatial domain. Hence one needs to sample the Green's function given in Fig. 3 at a period of less than 0.05 along $t$ so that the fine features of the function can be captured in the approximation. The choice of $T_{02}$ is another parameter that competes with the period of samples, because a large $T_{02}$ corresponds to large number of samples and translates into a longer CPU time. Fortunately, for the example given in Fig. 3, the Green's function decays quite rapidly in the spectral domain. Hence it is sufficient to sample the Green's function only as far as $T_{\mathrm{o} 2}=5$, which requires 200 samples if $\Delta t$ is chosen to equal 0.025 . The spatial-domain Green's function is obtained via the GPOF method using the above approximation parameters $\left(T_{\mathrm{o} 2}=5\right.$, number of samples $=201$, number of exponentials $=13$ ) and compared to the result obtained from the numerical integration, which are labeled as Apprx. and Exact, respectively, in Fig. 4. Although, as it 


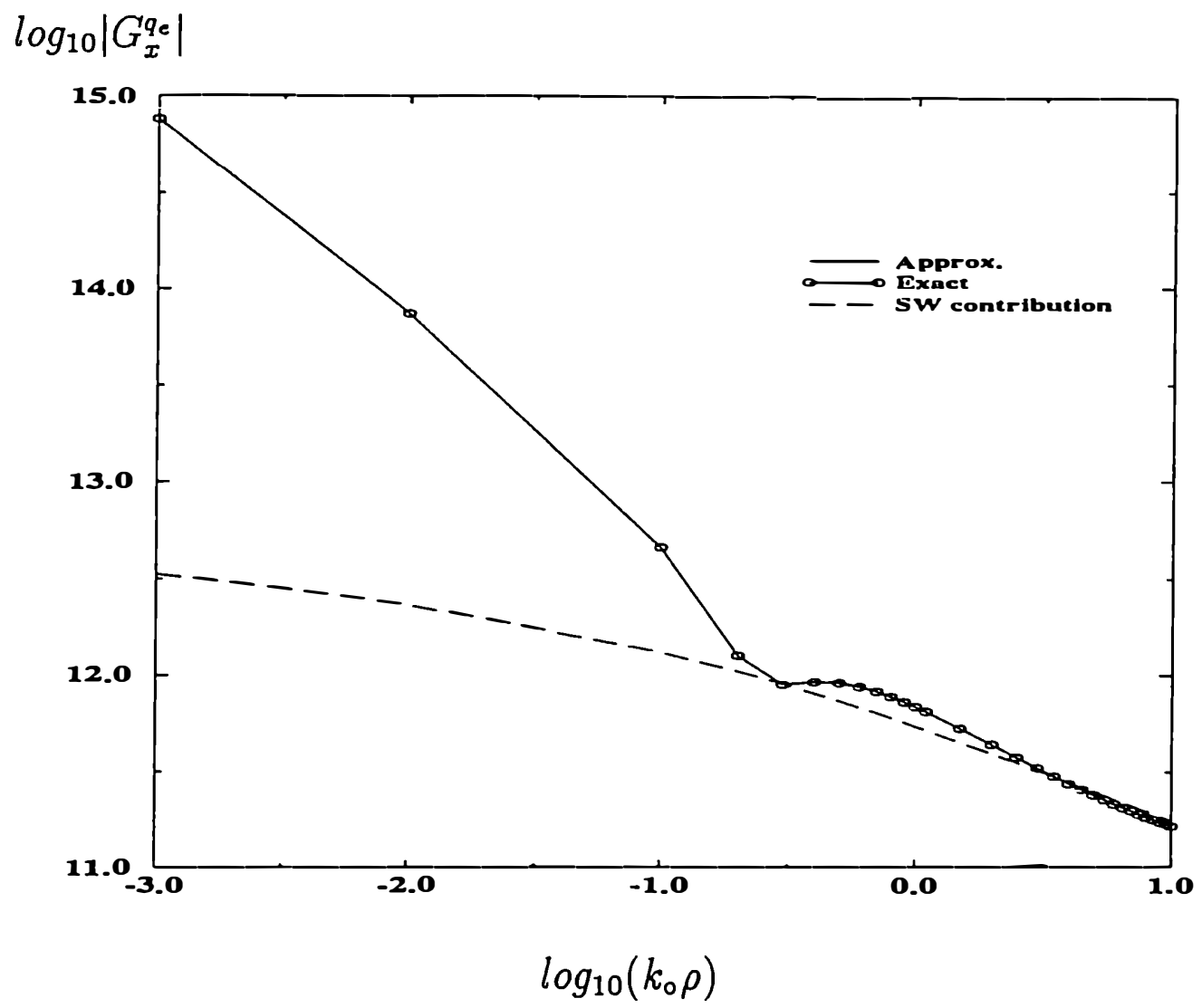

Figure 4: The magnitude of the Green's function for the scalar potential and the surface wave contribution. 1st layer- PEC; 2 nd layer- $\epsilon_{r 2}=12.5, d_{2}=0.03 \mathrm{~cm}$; 3rd layer- $\epsilon_{r 3}=2.1, d_{3}=0.07 \mathrm{~cm} ; 4$ th layer- free-space, freq $=30 \mathrm{GHz}$.

was mentioned above, the SWPs are not extracted from the spectral-domain Green's function prior to the exponential approximation, the contribution of the SWPs is also shown for the purpose of comparison. One can draw the conclusion that the exponential approximation algorithm (GPOF) works well within the influence range of the SWPs. Beyond that an asymptotic approximation together with the surface-wave contribution can be used to approximate the spatial-domain Green's functions ${ }^{35,}{ }^{36}$.

Unfortunately, not all the Green's functions have rapidly decaying spectral-domain behavior, of the type in the example given above in Fig. 3. For example, the spectraldomain Green's function for the vertical component of the vector potential due to a HED, $\tilde{G}_{z x}^{A} / j k_{x}=\tilde{G}_{z y}^{A} / j k_{y}(7)$, does not decay as fast and, moreover, has a relatively sharp peak which requires sampling almost as frequently as that of the example given in Fig. 3 (see Fig. 5). To demonstrate the effect of the choice of approximation parameters, the Green's function $\int G_{z x}^{A} d x\left(=\mathcal{F}^{-1}\left\{\tilde{G}_{z x}^{A} / j k_{x}\right\}\right)$ is given for the same approximation parameters as those of the above example $\left(T_{02}=5\right.$, number of samples $=201$ ) and compared to the results obtained by the numerical integration of the spectral-domain representation of the Green's function and to the results obtained by using different approximation parameters in Fig. 6. We observe that the approximated Green's functions do not agree well with the exact solution for small values of $\rho$. This is because the spectral-domain Green's function is not sampled far enough 


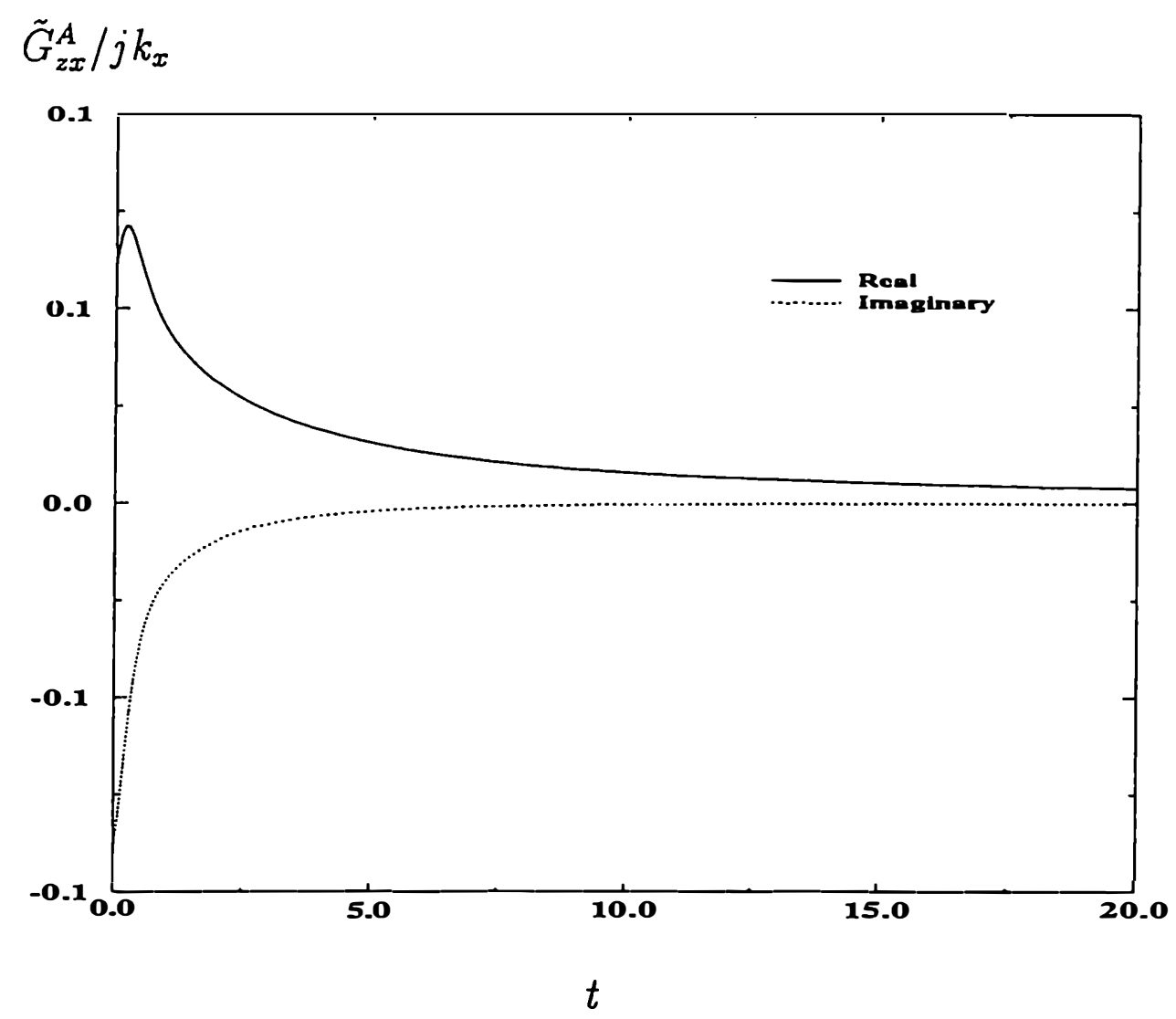

Figure 5: The magnitude of the spectral-domain Green's function $\tilde{G}_{z x}^{A} / j k_{x}$ along the path $C_{a p 2}$. 1st layer- PEC; 2nd layer- $\epsilon_{r 2}=12.5, d_{2}=0.03 \mathrm{~cm} ; 3$ rd layer- $\epsilon_{r 3}=2.1$, $d_{3}=0.07 \mathrm{~cm} ; 4$ th layer- free-space, freq $=30 \mathrm{GHz}$.

to get an accurate near-field distribution. However, if the value of $T_{\mathrm{o} 2}$ is increased, the agreement between the approximated and exact Green's functions is improved at the expense of the computation time provided that the frequency of sampling is kept constant.

From the above discussion, it can be concluded that the one-level approximation approach cannot be made fully robust and suitable for the development of CAD software. As was mentioned above, this is because it requires the users to first investigate the spectral-domain behavior of the Green's function and then perform a few iterations to find the best possible combination of the approximation parameters. To circumvent these difficulties, a two-level approximation scheme has been developed in conjunction with the use of the GPOF method and its details are given in the following section.

\subsubsection{Two-Level Approach}

To alleviate the necessity of investigating the spectral-domain Green's functions in advance and the difficulties caused by the trade-off between the sampling range $T_{\mathrm{o} 2}$ and the sampling period, the approximation is performed in two levels. The first part of the approximation is carried out along the path $C_{a p 1}$, and the second along the path $C_{a p 2}$, as shown in Fig. 2. Note that the second part of the approximation 


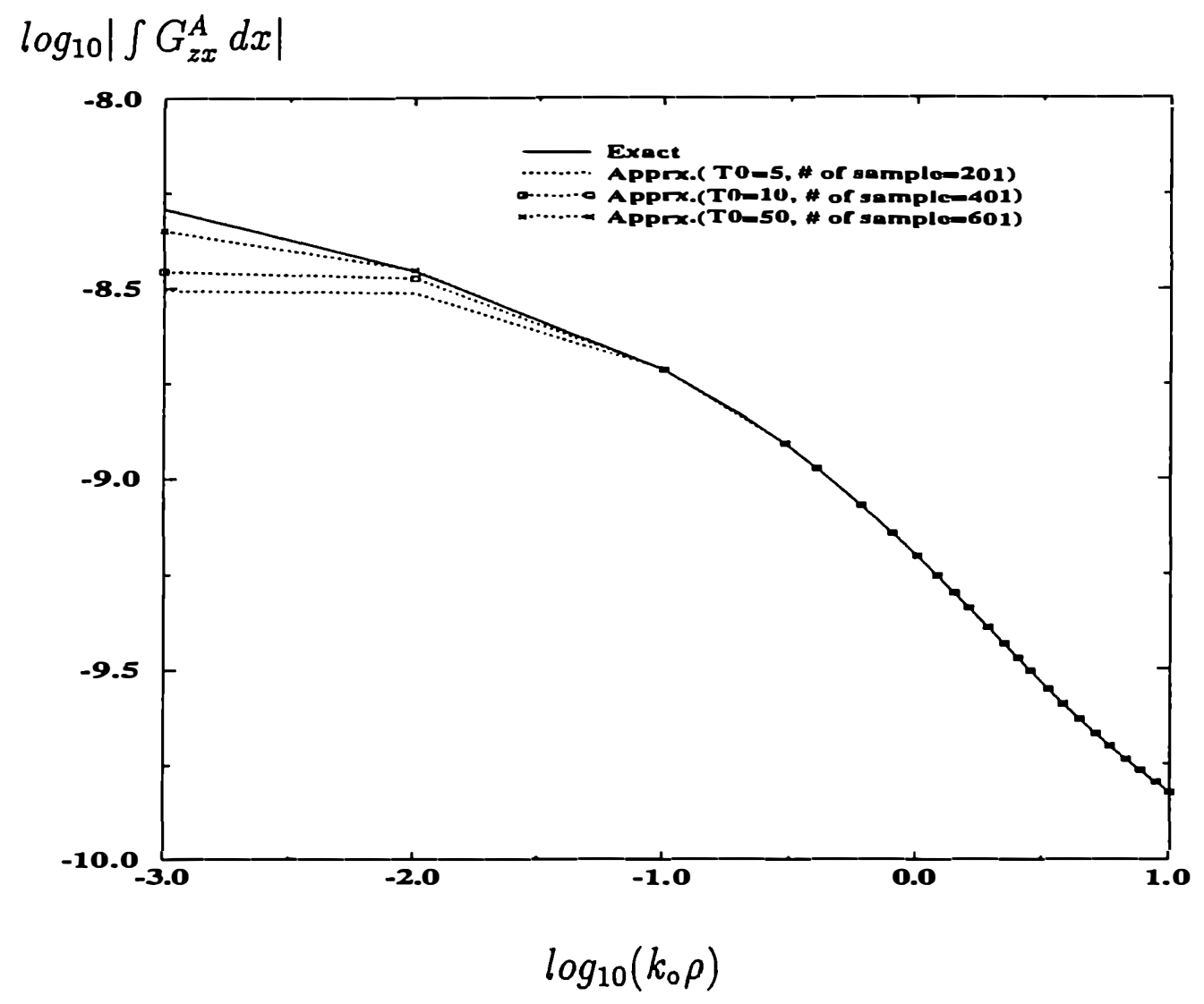

Figure 6: The magnitude of the Green's function for the vector potential $\int G_{z x}^{A} d x$. 1st layer- PEC; 2nd layer- $\epsilon_{r 2}=12.5, d_{2}=0.03 \mathrm{~cm}$; 3rd layer- $\epsilon_{r 3}=2.1, d_{3}=0.07 \mathrm{~cm} ; 4$ th layer- free-space, freq $=30 \mathrm{GHz}$.

is the same as the one-level approximation scheme described in the previous section, except that now the value of $T_{\mathrm{o} 2}\left(k_{\rho_{\max 2}}=k\left[1+T_{\mathrm{o} 2}^{2}\right]^{1 / 2}\right)$ can be set in advance such that $k_{\rho_{\max 2}} \geq k_{m}$ where $k_{m}$ is the maximum value of the wavenumber involved in the geometry.

To illustrate the procedure of the two-level approximation, we will first outline the necessary steps and then provide some of the details. The steps are:

- Choose $T_{\circ 2}$ such that $k_{\rho_{\max 2}} \geq k_{m}$ : For example, since GaAs is the highest dielectric constant layer $\left(\epsilon_{r}(G a A s)=12.5\right)$, then $k_{m}=\sqrt{12.5} k_{\mathrm{o}}$, and $T_{\mathrm{o} 2}$ can be safely chosen to be 5 .

- Choose $T_{01}$, i.e., $k_{\rho_{\max 1}}=k\left[1+\left(T_{01}+T_{02}\right)^{2}\right]^{1 / 2}$, and the number of samples in the range $\left[k_{\rho_{\max 2}}, k_{\rho_{\max } 1}\right]$. The choice of $T_{01}$ is not very critical as long as one chooses $k_{\rho_{\max 1}}$ large enough to pick up the behavior of the spectral-domain Green's function for large $k_{\rho}$. Also, since the spectral-domain behaviors of the Green's functions are always smooth beyond $k_{\rho_{\max 2}}$, it is not necessary to have a large number of sampies on $\left[k_{\rho_{\max 2}}, k_{\rho_{\max 1}}\right]$. Typical values could be 200 for $T_{01}$ and 200 for the number of samples.

- Sample the function along the path $C_{n p 1}$ and approximate it by using the GPOF 
method: Sampling along the path $C_{a p 1}$ can be performed by varying $t$ between 0 and $T_{01}$ uniformly in $k_{z}=-j k\left[T_{02}+t\right]$.

- Subtract the function approximated for the range of $k_{\rho} \in\left[k_{\rho_{\max 2}}, k_{\rho_{\max 1}}\right]$ from the original function: The remaining function will be non-zero over a small range of $k_{\rho}\left(\in\left[0, k_{\rho_{\max }}\right]\right)$ so that one can pick up the fine features of this function without employing a relatively large number of sampling points.

- Sample the remaining function along the path $C_{a p 2}$ uniformly and approximate it by using the GPOF method: Sampling along the path $C_{a p 2}$ can be performed by varying $t$ between 0 and $T_{\mathrm{o} 2}$ uniformly in $k_{z}=k\left[-j t+\left(1-t / T_{\mathrm{o} 2}\right)\right]$.

The parameters that must be fixed by the user in advance are the limits of the sampling ranges $T_{01}$ and $T_{02}$ for the first and the second parts of the approximation, respectively, and the number of samples along the paths $C_{a p 1}$ and $C_{a p 2}$, which respectively correspond to the first and second parts of the approximation. Although it appears the number of parameters that are to be decided by the user is greater than for the one-level approximation, these parameters need be determined only once for the class of geometries that are of interest. Also, they are used for the approximation of any component of the dyadic Green's function and for any geometrical constants. Finally, the choice of these parameters do not require an investigation of the function to be approximated in advance because they can be chosen for the possible limits of the geometrical constants.

To demonstrate the robustness of the technique, the choice of the parameters and the application of the above procedure, the Green's function $\int G_{z x}^{A} d x$ is obtained for the same geometry as considered in Section 2.2.1. Let us first write the parametric equations describing the paths $C_{a p 1}$ and $C_{a p 2}$ for the first and second parts of the approximation, respectively

$$
\begin{array}{ll}
\text { For } C_{a p 1} & k_{z_{i}}=-j k_{i}\left[T_{\mathrm{o} 2}+t\right] \quad 0 \leq t \leq T_{\mathrm{o} 1} \\
\text { For } C_{a p 2} & k_{z_{i}}=k_{i}\left[-j t+\left(1-\frac{t}{T_{02}}\right)\right] \quad 0 \leq t \leq T_{\mathrm{o} 2}
\end{array}
$$

where $t$ is the running variable sampled uniformly on the corresponding range. Then, the above procedure is followed step by step as:

- $T_{\mathrm{o} 2}=5$ is chosen, for which $k_{\rho_{\max 2}}=k_{i}\left[1+T_{\mathrm{o} 2}^{2}\right]^{1 / 2}>k_{m}=\sqrt{12.5} k_{\circ}$

- $T_{\mathrm{o} 1}=400$ is chosen to ensure that the behavior of $\tilde{G}_{z x}^{A} / j k_{x}$ for large $k_{\rho}$ is captured. This choice for $T_{\text {o1 }}$ is not critical; for instance, a value of 300 or 500 could have been chosen instead. Since there is no fine feature to be modeled in this range, i.e., since the function is smooth, one can keep the range large without having to use a large number of samples. Therefore, the number of samples is chosen to be 50 . 
- $\tilde{G}_{z x}^{A} / j k_{x}$ is sampled along the path $C_{a p 1}$ and the GPOF method is applied.

$$
\begin{aligned}
f\left(k_{\rho}\right)\left(\cong \frac{\tilde{G}_{z x}^{A}}{j k_{x}} \text { for } \quad k_{\rho} \in\left[k_{\rho_{\max }}, k_{\rho_{\max }}\right]\right) & =\sum_{n=1}^{N_{1}} b_{1 n} e^{\beta_{1 n} t} \\
& =\sum_{n=1}^{N_{1}} a_{1 n} e^{-\alpha_{1 n} k_{z_{i}}} \\
\alpha_{1 n}=\frac{\beta_{1 n}}{j k_{i}} ; \quad a_{1 n}=b_{1 n} e^{-j k_{i} \alpha_{1 n} T_{02}} &
\end{aligned}
$$

where $b_{1 n}$ and $\beta_{1 n}$ are the coefficients and exponents obtained from the GPOF method, and $N_{1}$ is the number of exponentials used in this approximation. The choice of the number of exponentials is based upon the number of significant singular values obtained in an intermediate step of the application of the GPOF method. For this specific problem, five exponentials are chosen to approximate the Green's function over the range of $k_{\rho} \in\left[k_{\rho_{\max 2}}, k_{\rho_{\max }}\right]$. It is necessary to transform the coefficients $b_{1 n}$ and the exponents $\beta_{1 n}$ is necessary to cast the approximating function into a form suitable for the application of the Sommerfeld identity (29). This, in turn, implies that the approximating function must be an exponential representation in terms of $k_{z_{\mathrm{i}}}$. Hence, $a_{1 n}$ and $\alpha_{1 n}$ are obtained in terms of $b_{1 n}$ and $\beta_{1 n}$ in (34).

- The approximating function $f\left(k_{\rho}\right)$ is subtracted from the original function $\tilde{G}_{z x}^{A} / j k_{x}$, which guarantees the remaining function to be negligible beyond $k_{\rho \max 2}$.

$$
\begin{array}{r}
G=\frac{1}{4 \pi} \int_{C_{a p 2}} d k_{\rho} k_{\rho} H_{o}^{(2)}\left(k_{\rho} \rho\right)\left[\frac{\tilde{G}_{z x}^{A}\left(k_{\rho}\right)}{j k_{x}}-f\left(k_{\rho}\right)\right]+ \\
\frac{1}{4 \pi} \int_{C_{a p 2}+C_{a p 1}} d k_{\rho} k_{\rho} H_{o}^{(2)}\left(k_{\rho} \rho\right) f\left(k_{\rho}\right)
\end{array}
$$

Note that the first integral is evaluated along the path $C_{a p 2}$ because the integrand is negligible on $C_{a p 1}$, but the second integral is evaluated along $C_{a p 2}+C_{a p 1}$. Therefore, the Sommerfeld identity (29) can be applied to the integrals in (35).

The remaining function is sampled along the path $C_{a p 2}$ with 100 samples. Since the maximum range for the sampling $\left(k_{\rho_{\max 2}}\right)$ is rather small compared to that of the one-level approximation scheme, the frequency of sampling can be made quite high without substantially increasing the number of samples. For all practical purposes (including the worst case situation) the choice of 200 as the number of samples is more than sufficient to get a good approximation.

$$
\begin{gathered}
\frac{\tilde{G}_{z x}^{A}}{j k_{x}}-f\left(k_{\rho}\right) \cong \sum_{n=1}^{N_{2}} b_{2 n} e^{\beta_{2 n} t}=\sum_{n=1}^{N_{2}} a_{2 n} e^{-\alpha_{2 n} k_{z_{i}}} \\
\alpha_{2 n}=\frac{\beta_{2 n} T_{02}}{k_{i}\left(1+j T_{02}\right)} ; \quad a_{2 n}=b_{2 n} e^{k_{i} \alpha_{2 n}}
\end{gathered}
$$


where $b_{2 n}$ and $\beta_{2 n}$ are the coefficients and exponents of the exponentials of $t$ obtained from the application of the GPOF method, and $a_{2 n}$ and $\alpha_{2 n}$ are the coefficients and exponents of the exponentials of $k_{z_{i}}$. The number of exponentials $N_{2}$ in this part of the approximation is chosen to be 8 , again by the number of significant singular values.

- By following the previous steps, the spectral-domain Green's function can be written as

$$
\tilde{G} \cong \frac{1}{j 2 k_{z_{i}}}\left[\sum_{n=1}^{N_{1}} a_{1 n} e^{-\alpha_{1 n} k_{z_{i}}}+\sum_{n=1}^{N_{2}} a_{2 n} e^{-\alpha_{2 n} k_{z_{i}}}\right]
$$

Also the spatial-domain Green's function can be cast into a closed-form by substituting this approximation of the spectral-domain Green's function into Eq. (28), and then employing the Sommerfeld identity Eq. (29) for each term to yield

$$
G \cong \sum_{n=1}^{N_{1}} a_{1 n} \frac{e^{-j k_{i} r_{1 n}}}{r_{1 n}}+\sum_{n=1}^{N_{2}} a_{2 n} \frac{e^{-j k_{i} r_{2 n}}}{r_{2 n}}
$$

where $r_{1 n}=\sqrt{x^{2}+y^{2}-\alpha_{1 n}^{2}}$ and $r_{2 n}=\sqrt{x^{2}+y^{2}-\alpha_{2 n}^{2}}$ are the complex distances.

In summary, the approximation parameters chosen here for the first part of the approximation are: $T_{\mathrm{o} 1}=400 ; T_{02}=5$; number of samples $=50$; and, number of exponentials $=5$. Likewise, for the second part of the approximation these parameters are: number of samples $=100$; and, number of exponentials $=8$. Note that the total number of exponentials used in this approximation is 13. The Green's function derived by employing the above procedure is plotted in Fig. 7, along with the data obtained from direct numerical evaluation of the Sommerfeld-type integral (exact). Also plotted in Fig. 7 are the results derived from the one-level approximation approach with the following parameters of approximation: $T_{0}=200$; number of samples $=400$; and the number of exponentials=13. Note that the values of the parameters used in the onelevel approximation are chosen to reduce the computation time without sacrificing too much accuracy. However, those of the two-level approximation are typical values and the number of samples for the second part of the approximation can even be reduced to 50 with little or no change in the results. The CPU times on the SPARCstation 10/41 for two approximation techniques for the above example are compared when the same number $(=13)$ of total exponentials are used for different approximation parameters. The results are presented in the tabular format below:

$\begin{array}{ccc}\text { Approximation } & \text { Approximation Parameters } & \text { CPU time }(\mathrm{sec}) \\ \text { one-level } & T_{\mathrm{o}}=200, N_{s}=400 & 198.0 \\ \text { one-lcvel } & T_{\mathrm{o}}=200, N_{s}=500 & 382.0 \\ \text { two-level } & T_{\mathrm{o} 1}=400, N_{s_{1}}=50 T_{\mathrm{o} 2}=5, N_{s_{2}}=50 & 1.2 \\ \text { two-level } & T_{\mathrm{o} 1}=400, N_{s_{1}}=50 T_{\mathrm{o} 2}=5, N_{s_{2}}=100 & 3.5\end{array}$




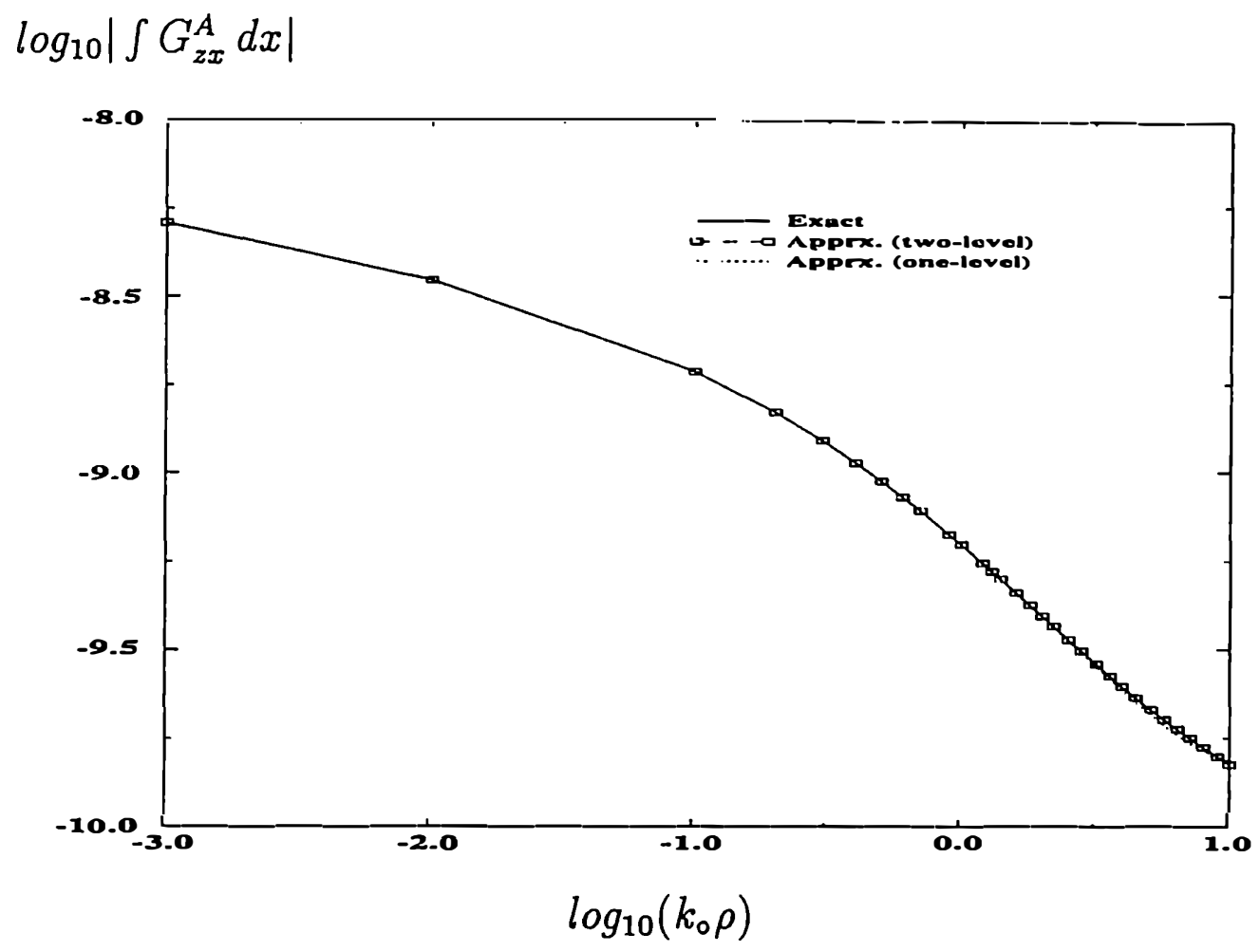

Figure 7: The magnitude of the Green's function for the vector potential $\int G_{z x}^{A} d x$. 1st layer- PEC; 2nd layer- $\epsilon_{r 2}=12.5, d_{2}=0.03 \mathrm{~cm}$; 3 rd layer- $\epsilon_{r 3}=2.1, d_{3}=0.07 \mathrm{~cm} ; 4$ th layer- free-space, freq $=30 \mathrm{GHz}$.

where $N_{s}$ is the number of samples in the one-level approximation scheme while $N_{s 1}$ and $N_{s 2}$ are the number of samples of the first and second parts of the approximation, respectively, in the two-level approximation approach. It is obvious that the two-level approximation approach improves the computational efficiency significantly.

The robustness of the two-level approach can be demonstrated by casting the other Green's functions into closed forms with the use of the same approximation parameters as those employed for $\int G_{z x}^{A} d x$, viz., $T_{\mathrm{o} 1}=400, N_{s_{1}}=50 T_{\mathrm{o} 2}=5$, $N_{s_{2}}=100$. The normalized Green's functions of the vector and scalar potentials due to HED and VED sources are obtained $\left(4 \pi G_{x x}^{A} / \mu_{3}, 4 \pi \epsilon_{3} G_{x}^{q}, 4 \pi G_{z z}^{A} / \mu_{3}\right.$ and $\left.4 \pi \epsilon_{3} G_{z}^{q}\right)$ following the two-level approach (Apprx.) and evaluating the Sommerfeld integrals numerically (Exact). These Green's function plots are shown in Figs. 8 and 9. This numerical experimentation shows that the same set of approximation parameters can be used for any Green's function, i.e., neither an advance investigation of the Green's function nor any trial steps are needed in this procedure.

The assessment of the robustness of the proposed approach also requires a study of the sensitivity of the approximation parameters to the geometrical constants and the frequency. Hence the Green's functions for the vector and the scalar potentials are obtained in closed forms for the same geometrical constants and for the same approximation parameters used above, but the frequency of operation is changed to $1 \mathrm{GHz}, 10 \mathrm{GHz}$ and $100 \mathrm{GHz}$. This is equivalent, in effect, to changing the geometrical constants (see Figs. 10 and 11). It is observed that the agreements between the exact 


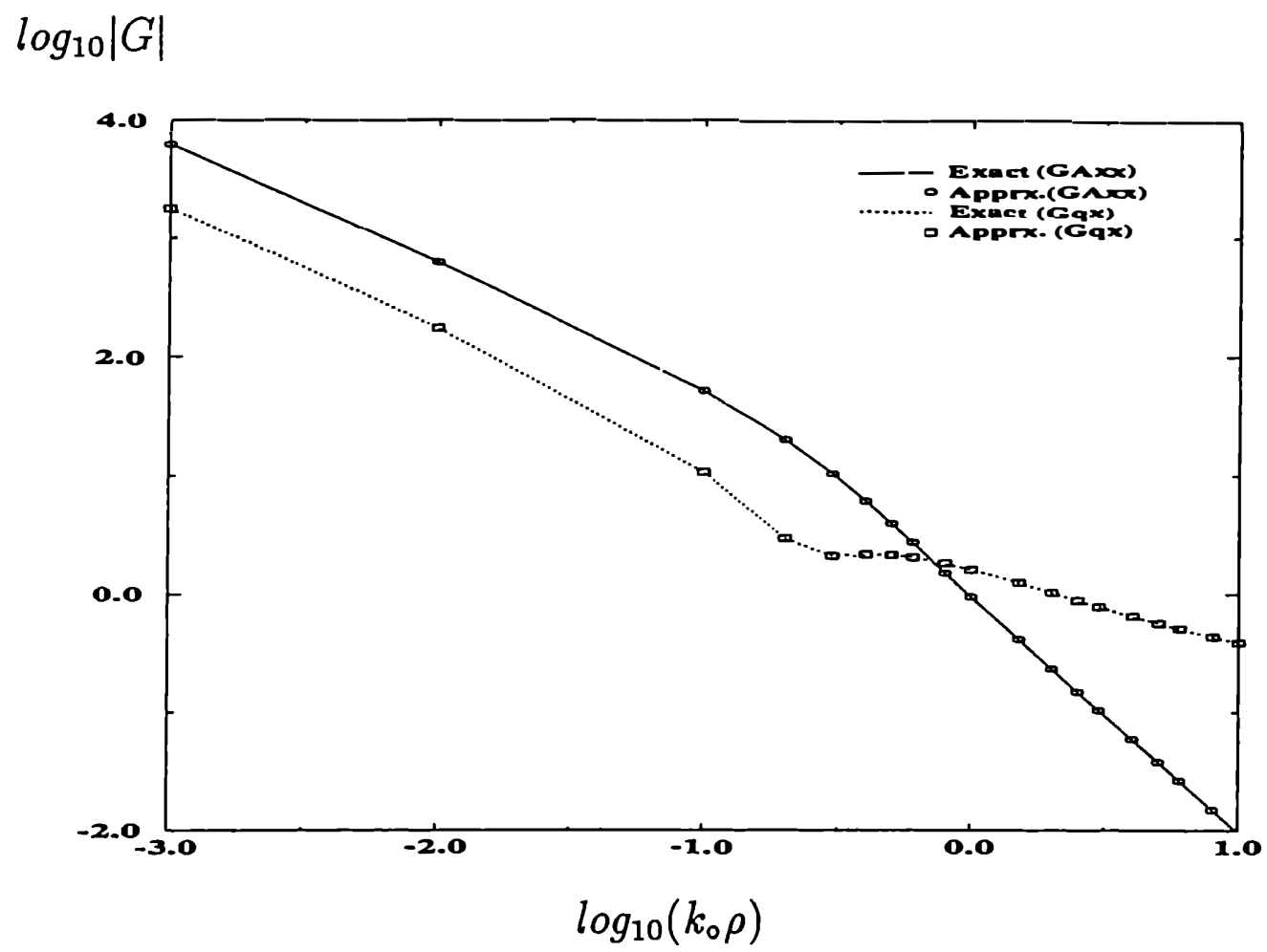

Figure 8: The magnitude of the normalized Green's functions $4 \pi G_{x x}^{A} / \mu_{3}, 4 \pi \epsilon_{3} G_{x}^{q}$. 1st layer- PEC; 2nd layer- $\epsilon_{r 2}=12.5, d_{2}=0.03 \mathrm{~cm} ; 3$ rd layer- $\epsilon_{r 3}=2.1, d_{3}=0.07 \mathrm{~cm} ; 4$ th layer- free-space, freq $=30 \mathrm{GHz}$.

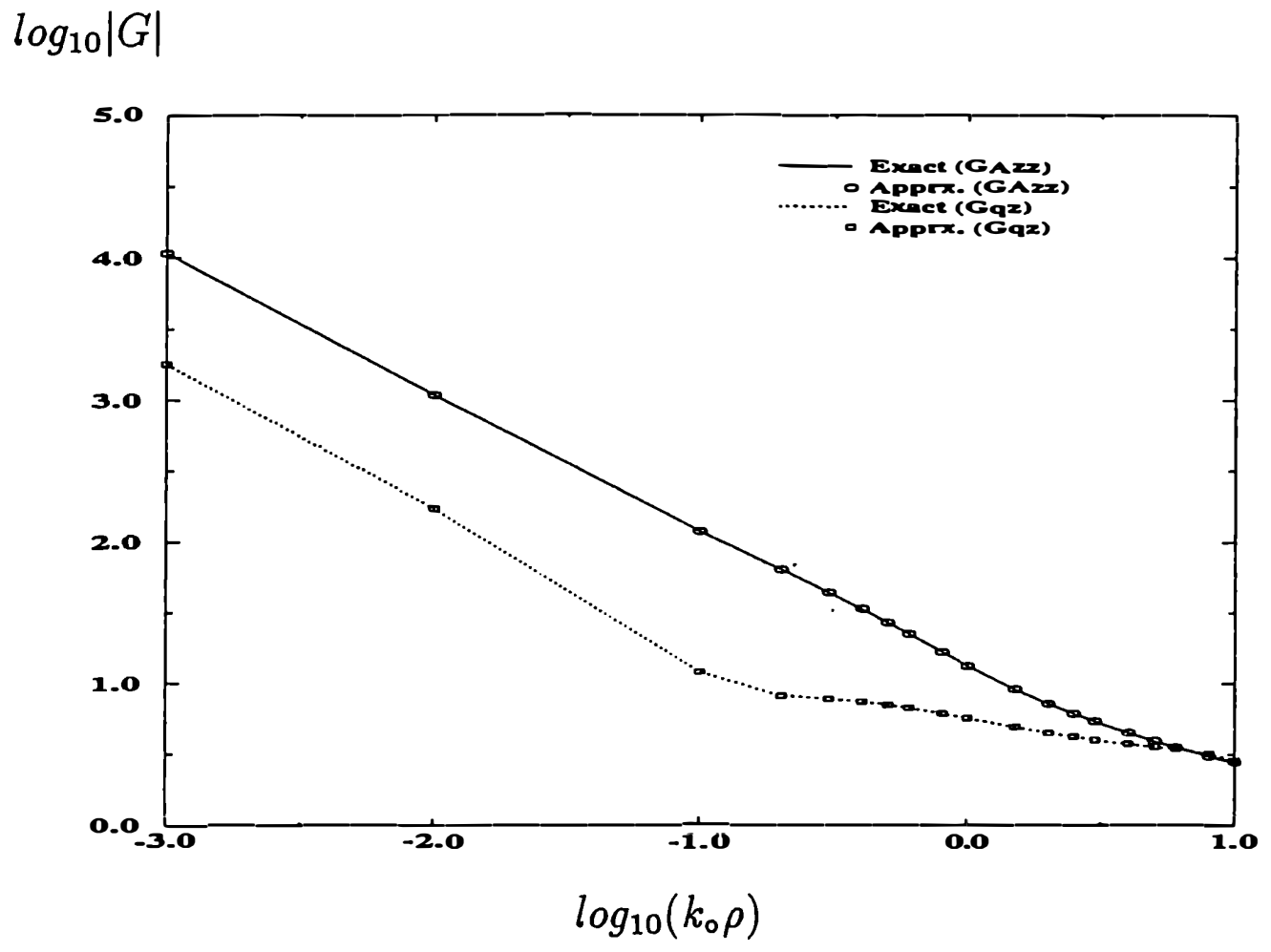

Figure 9: The magnitude of the normalized Green's functions $4 \pi G_{z z}^{A} / \mu_{3}, 4 \pi \epsilon_{3} G_{z}^{q}$. 1st layer- PEC; 2nd layer- $\epsilon_{r 2}=12.5, d_{2}=0.03 \mathrm{~cm}$; 3rd layer- $\epsilon_{r 3}=2.1, d_{3}=0.07 \mathrm{~cm} ; 4$ th layer- free-space, freq $=30 \mathrm{GHz}$. 


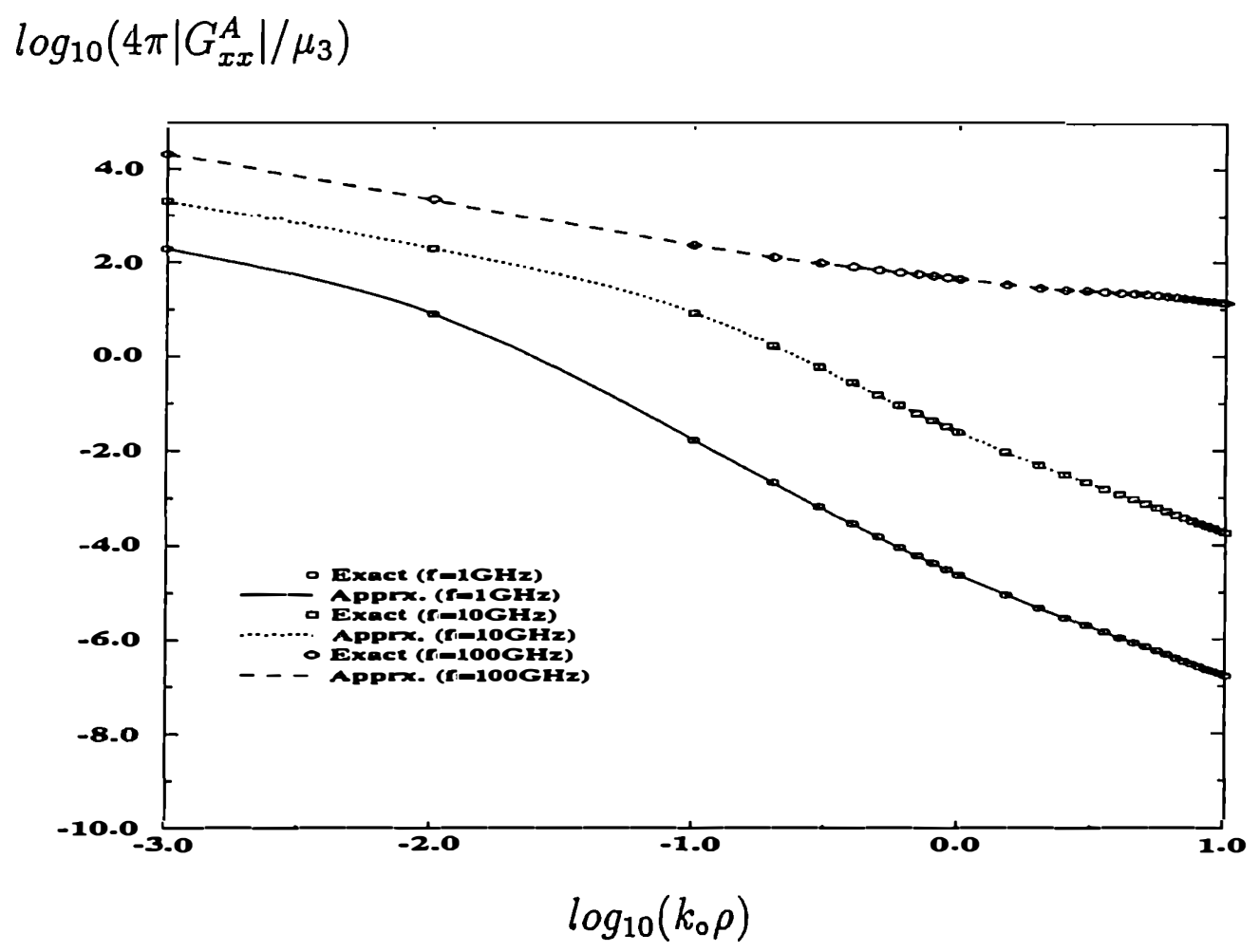

Figure 10: The magnitude of the normalized Green's function $4 \pi G_{x x}^{A} / \mu_{3}$. 1st layerPEC; 2nd layer- $\epsilon_{r 2}=12.5, d_{2}=0.03 \mathrm{~cm}$; 3rd layer- $\epsilon_{r 3}=2.1, d_{3}=0.07 \mathrm{~cm}$; 4th layerfree-space.

and approximate sets of data remain extremely good.

\section{MoM Applications of the Closed-Form Green's Functions}

After having introduced a robust and computationally efficient approach for the derivation of the closed-form Green's functions in the previous section, their use in the method of moments formulation is discussed in this section. Eliminating the Sommerfeld integral in the application of the spatial-domain MoM results in two-dimensional integrals over finite ranges, and, consequently, the computational efficiency improves significantly. However, one still needs to evaluate the remaining double integrals numerically, which is the limiting factor for matrix-fill time in the application of the spatial-domain MoM for a printed geometry. In this section, we will demonstrate that the use of the complex exponentials in place of the spatial-domain Green's functions further improves the matrix-fill time because it facilitates the analytical evaluation of the remaining double integrals. Thus, a substantial improvement in the matrix-fill time is achieved by eliminating all of the numerical integrations involved in the application of the spatial-domain MoM to the solution of the Mixed Potential Integral Equation. 


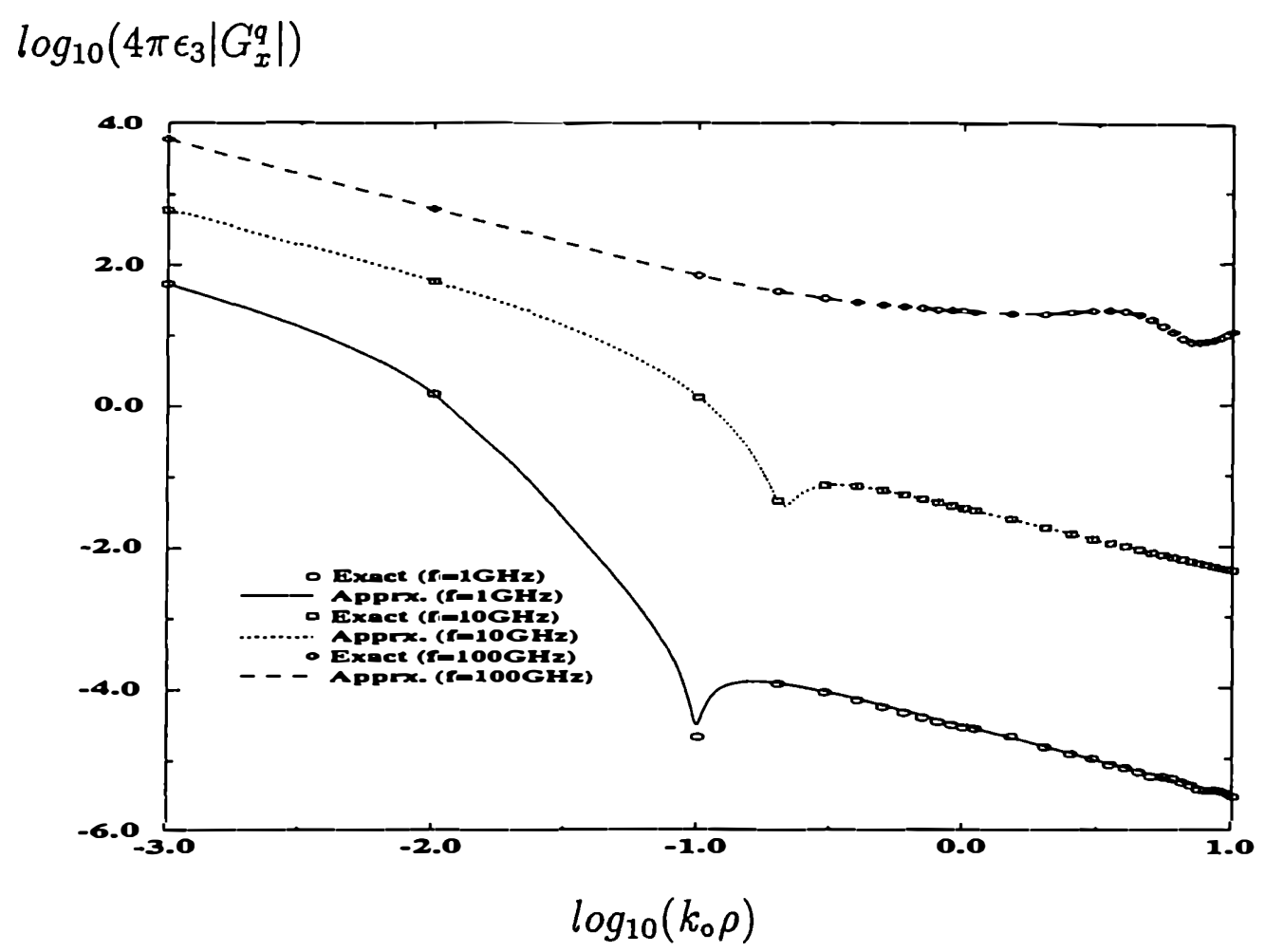

Figure 11: The magnitude of the normalized Green's function $4 \pi \epsilon_{3} G_{x}^{q}$. 1st layerPEC; 2nd layer- $\epsilon_{r 2}=12.5, d_{2}=0.03 \mathrm{~cm}$; 3rd layer- $\epsilon_{r 3}=2.1, d_{3}=0.07 \mathrm{~cm}$; 4 th layerfree-space.

\subsection{Formulation for the Spatial-Domain MoM}

Before going into the details of how the closed-form Green's functions are employed in the MoM formulations, it would be instructive to present the formulation for the spatial-domain MoM for 2.5D geometries in a multilayer environment. For the sake of illustration, a typical 2.5D geometry is depicted in Fig. 12 and the MoM formulation is demonstrated for this geometry with no loss of generality. Note that the geometry is multilayer and has vertical connections, e.g., shorting pins, as well as two-dimensional printed conductors. All of the layers and the ground plane are assumed to be infinitely wide in the horizontal plane, and the conductors are assumed to be lossless and infinitesimally thin.

The tangential components of the electric field on the plane of the patch and on the shorting pins can be written in terms of the surface current density, $\mathbf{J}$, and the associated Green's functions as follows:

$$
\begin{gathered}
E_{x}=-j w G_{x x}^{A} * J_{x}+\frac{1}{j w} \frac{\partial}{\partial x}\left(G^{q_{e}} * \nabla \cdot \mathbf{J}\right) \\
E_{y}=-j w G_{y y}^{A} * J_{y}+\frac{1}{j w} \frac{\partial}{\partial y}\left(G^{q_{e}} * \nabla \cdot \mathbf{J}\right) \\
E_{z}=-j w G_{z x}^{A} * J_{x}-j w G_{z y}^{A} * J_{y}-j w G_{z z}^{A} * J_{z}+\frac{1}{j w} \frac{\partial}{\partial z}\left(G^{q_{e}} * \nabla \cdot \mathbf{J}\right)
\end{gathered}
$$




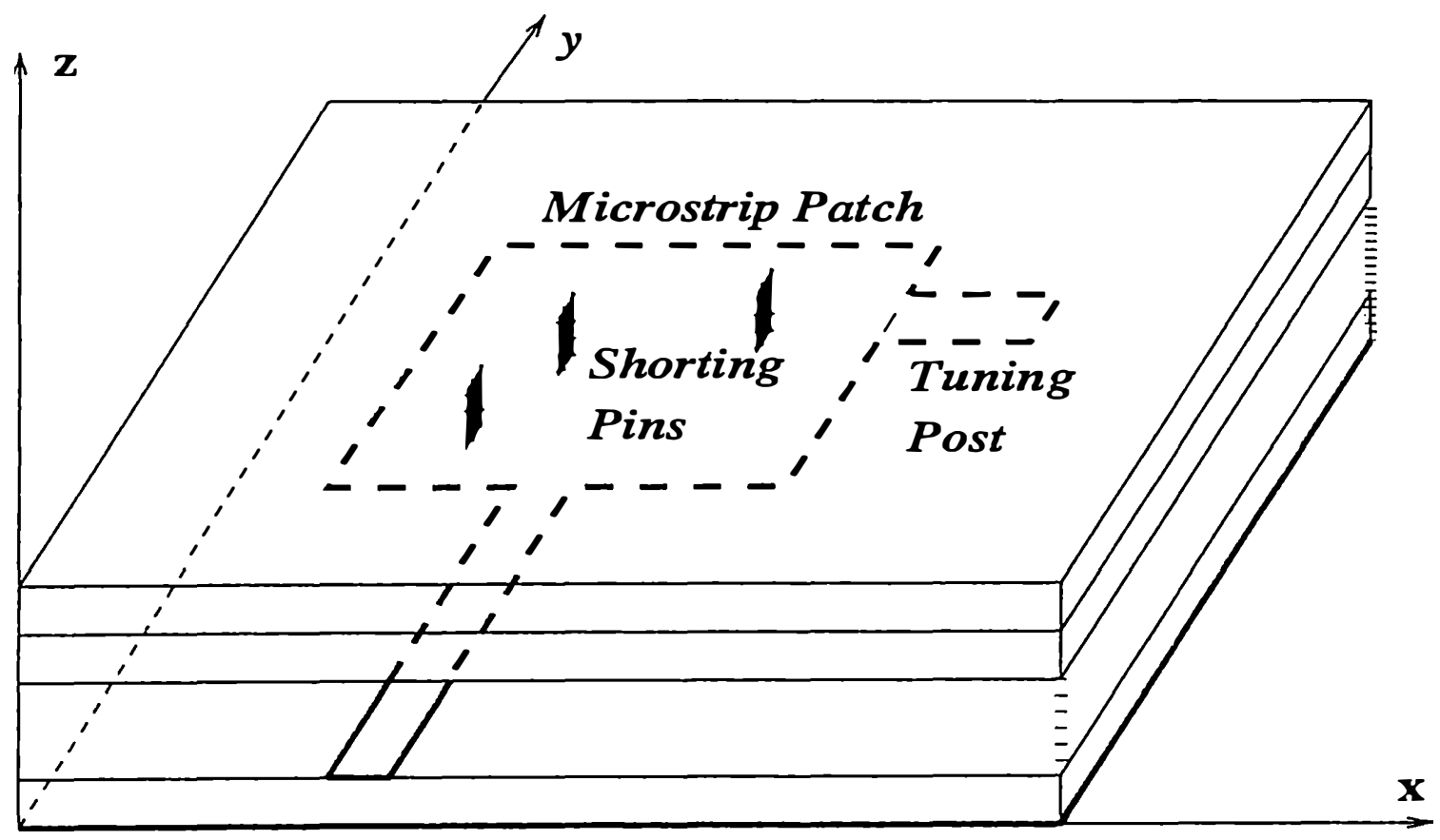

Ground Plane

Figure 12: A typical 2.5D geometry in a multilayer medium.

where * denotes convolution and $G_{x x}^{A}=G_{y y}^{A} . G_{i j}^{A}$ represents the $i$-directed vector potential at $\mathbf{r}$ due to an $j$-directed electric dipole of unit strength located at $\mathbf{r}^{\prime}$, while $G^{q_{e}}$ represents the scalar potential by a unit point charge associated with an electric dipole. Since the traditional form of the Green's functions are employed in the above formulation, the Green's function for the scalar potential is not unique for the HED and VED. Hence, the term involving the Green's function for the scalar potential, which is common in Eqs. (40) -(42), can be explicitly written as

$$
G^{q_{e}} * \nabla \cdot \mathbf{J}=G_{x}^{q_{e}} * \frac{\partial J_{x}}{\partial x}+G_{y}^{q_{e}} * \frac{\partial J_{y}}{\partial y}+G_{z}^{q_{e}} * \frac{\partial J_{z}}{\partial z}
$$

where $G_{x}^{q_{e}}=G_{y}^{q_{e}}$ for an HED and $G_{z}^{q_{e}}$ for an VED.

To solve for the surface current density $\mathbf{J}$ via the $\mathbf{M o M}, \mathbf{J}$ is expressed as a linear combination of the basis functions, which are chosen in this work to be rooftops for the $\mathrm{x}$ - and $\mathrm{y}$-components and pulses or rooftops for the z-component of the current density (see Fig. 13). Hence, the components of the current density are expressed as:

$$
\begin{aligned}
J_{x}(x, y) & =\sum_{m} \sum_{n} I_{x}^{(m n)} B_{x}^{(m n)}(x, y) \\
J_{y}(x, y) & =\sum_{m} \sum_{n} I_{y}^{(m n)} B_{y}^{(m n)}(x, y) \\
J_{z}(x, y, z) & =\sum_{l} I_{z}^{(l)} B_{z}^{(l)}(x, y, z)
\end{aligned}
$$




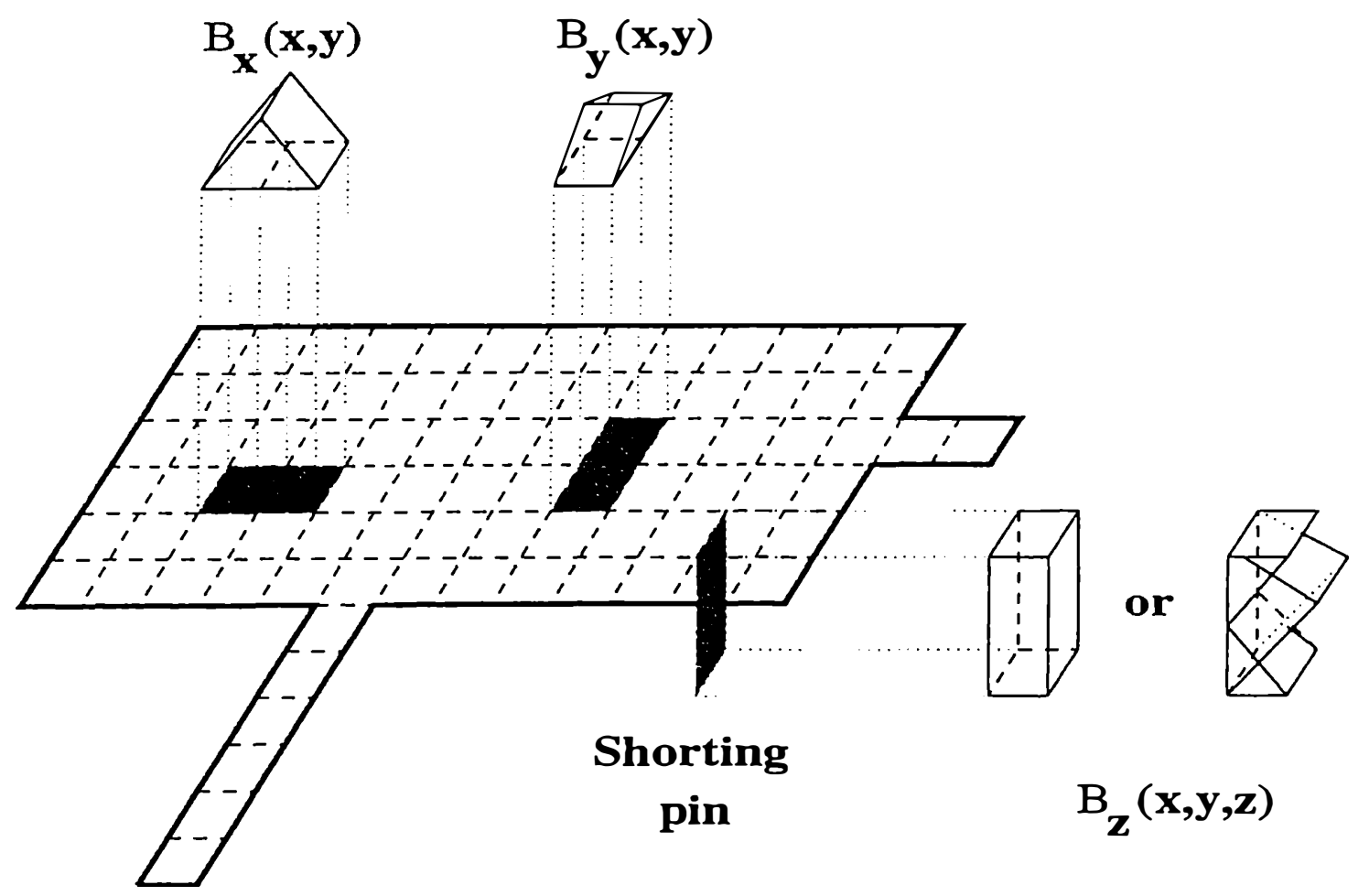

Figure 13: Basis functions representing the current density.

where $B_{x}^{(m n)}$ and $B_{y}^{(m n)}$ are rooftop functions, and $B_{z}^{(l)}$ is pulse or rooftop functions, $I_{x}^{(m n)}$ and $I_{y}^{(m n)}$ are the unknown coefficients of the basis functions at the $(m, n)$ th position on the subdivided horizontal conductor, and $I_{z}^{(l)}$ on the $l$-th shorting pin or the via hole. One of the basis function can be considered as that associated with the current source, of course with a known coefficient.

Following the substitution of Eqs. (44)-(46) into Eqs. (40)-(42), the boundary conditions on the tangential electric fields on the conductors are applied in integral sense by testing the resulting equations with some testing functions, say $T_{x}^{\left(m^{\prime} n^{\prime}\right)}$, $T_{y}^{\left(m^{\prime} n^{\prime}\right)}$ and $T_{z}^{\left(l^{\prime}\right)}$. This leads to a matrix equation for the unknown coefficients of the basis functions which reads

$$
\begin{gathered}
{[\mathrm{Z}][\mathrm{I}]=[\mathrm{V}]} \\
{\left[\begin{array}{lll}
Z_{x x}^{\left(m^{\prime} n^{\prime}, m n\right)} & Z_{x y}^{\left(m^{\prime} n^{\prime}, m n\right)} & Z_{x z}^{\left(m^{\prime} n^{\prime}, l\right)} \\
Z_{y x}^{\left(m^{\prime} n^{\prime}, m n\right)} & Z_{y y}^{\left(m^{\prime} n^{\prime}, m n\right)} & Z_{y z}^{\left(m^{\prime} n^{\prime}, l\right)} \\
Z_{z x}^{\left(l^{\prime}, m n\right)} & Z_{z y}^{\left(l^{\prime}, m n\right)} & Z_{z z}^{\left(l^{\prime}, l\right)}
\end{array}\right]\left[\begin{array}{l}
I_{x}^{(m n)} \\
I_{y}^{(m n)} \\
I_{z}^{(l)}
\end{array}\right]=\left[\begin{array}{l}
V_{x}^{\left(m^{\prime} n^{\prime}\right)} \\
V_{y}^{\left(m^{\prime} n^{\prime}\right)} \\
V_{z}^{\left(l^{\prime}\right)}
\end{array}\right]}
\end{gathered}
$$

where $Z$ s denote the mutual impedances between the testing and basis functions, and $V$ s represent the excitation voltages due to the current source. For the sake of brevity, the explicit forms of the impedances and voltages in Eq. (47) are not given here for this general case. 


\subsection{Closed-Form Grcen's Functions for MoM applications}

In Section 2, a general procedure for deriving the closed-form Green's functions for the vector and scalar potentials was detailed. However, a discussion of the technique for rendering these closed-form expressions numerically efficient when they are used in conjunction with the method of moments was not included. As mentioned in Section 2.2, the terms in the square brackets in Eqs. (6)-(15) should be sampled uniformly along $k_{z i}$ and approximated in terms of complex exponentials. However, in order to do this, it is necessary to fix the vertical coordinate variable $z$ and apply the GPOF approximation technique anew for each value of $z$. For geometries involving horizontal conductors only, this would not be a computationally inefficient procedure because the conductors would be located at constant $z$-planes. As an example, for a microstrip line along the $\mathrm{x}$-direction, the only MoM matrix element is $Z_{x \boldsymbol{x}}^{\left(m m^{\prime}\right)}$, and can be written explicitly as;

$$
Z_{x x}^{\left(m m^{\prime}\right)}=<T_{x}^{\left(m^{\prime}\right)}, G_{x x}^{A} * B_{x}^{(m)}>+\frac{1}{\omega^{2}}<T_{x}^{\left(m^{\prime}\right)}, \frac{\partial}{\partial x}\left[G_{x}^{q_{e}} * \frac{\partial B_{x}^{(m)}}{\partial x}\right]>
$$

where $G_{x x}^{A}$ and $G_{x}^{q_{e}}$ used in this formulation are approximated by GPOF for constant $z$ 's corresponding to the planes of the conductors in the geometry. However, for geometries with both vertical and horizontal conductors that support both $z$ and $x$-directed current components, typical MoM matrix elements have the forms

$$
\begin{aligned}
< & T_{z}^{\left(l^{\prime}\right)}, G_{z x}^{A} * B_{x}^{(m)}>, \quad<T_{z}^{\left(l^{\prime}\right)}, \frac{\partial}{\partial z}\left[G_{x}^{q_{e}} * \frac{\partial B_{x}^{(m)}}{\partial x}\right]> \\
& <T_{z}^{\left(l^{\prime}\right)}, G_{z z}^{A} * B_{z}^{(l)}>, \quad<T_{z}^{\left(l^{\prime}\right)}, \frac{\partial}{\partial z}\left[G_{z}^{q_{e}} * \frac{\partial B_{z}^{(l)}}{\partial z}\right]>
\end{aligned}
$$

where $<,>$ and * denote inner product and convolution, respectively. $G_{z x}^{A}, G_{z z}^{A}, G_{x}^{q_{e}}$ and $G_{z}^{q_{e}}$ need to be approximated at every observation point $z$, and/or source point $z^{\prime}$ values in the integration due to the testing and expansion processes along a vertical conductor. (Note that if we assume the origin is located at the bottom of the source layer for the application of the MoM, the coordinates used in the derivation of the Green's functions here can be transformed from $z$ to $z-z^{\prime}$, and $h$ to $z^{\prime}$ ). However, this would defeat the purpose of using the closed-form Green's functions in a MoM application. To circumvent the problem associated with the testing process, which corresponds to integration along $z$, the GPOF method can be applied to the complex coefficients of $e^{ \pm j k_{z_{i}} z}$ in all the Green's functions except $\tilde{G}_{x x}^{A, F}$. Hence, the $z$-dependence in the closed-form Green's functions becomes explicit and the testing procedure along the z-direction can be performed analytically for some testing functions like uniform and roof-top functions, which further improves the computational efficiency of using the closed-form Green's functions in conjunction with the method of moments.

Another technique, proposed herein, to overcome the above mentioned difficulties is to interchange the order of integration (49), provided that the basis and testing 
functions are so chosen that the involved integrals are uniformly convergent ${ }^{37}$, and carrying out the integration over $z$ analytically for spectral domain representation of the Green's function multiplied with the testing function. Next, the approximation method, GPOF, is applied to the resulting spectral domain function. For the inner product terms involving both the $z$ and $z^{\prime}$ integrations, these integrals can be performed analytically by using the procedure described above, and subsequently applying the GPOF algorithm. Note that the spectral representations of the Green's functions are exponential functions of $z$ and $z^{\prime}$, and this enables us to carry out the $z$ and $z^{\prime}$ integrations analytically in the spectral domain.

\subsection{Analytical Evaluation of the MoM Matrix Elements}

The formulation presented herein is applicable to general microstrip geometries in a multilayer medium where it is assumed that the layers extend to infinity in the transverse directions. However, to illustrate the main concept of the proposed method, the formulation is presented, without loss of generality, for a microstrip etch on a substrate for which only the longitudinal current is assumed to exist. To calculate

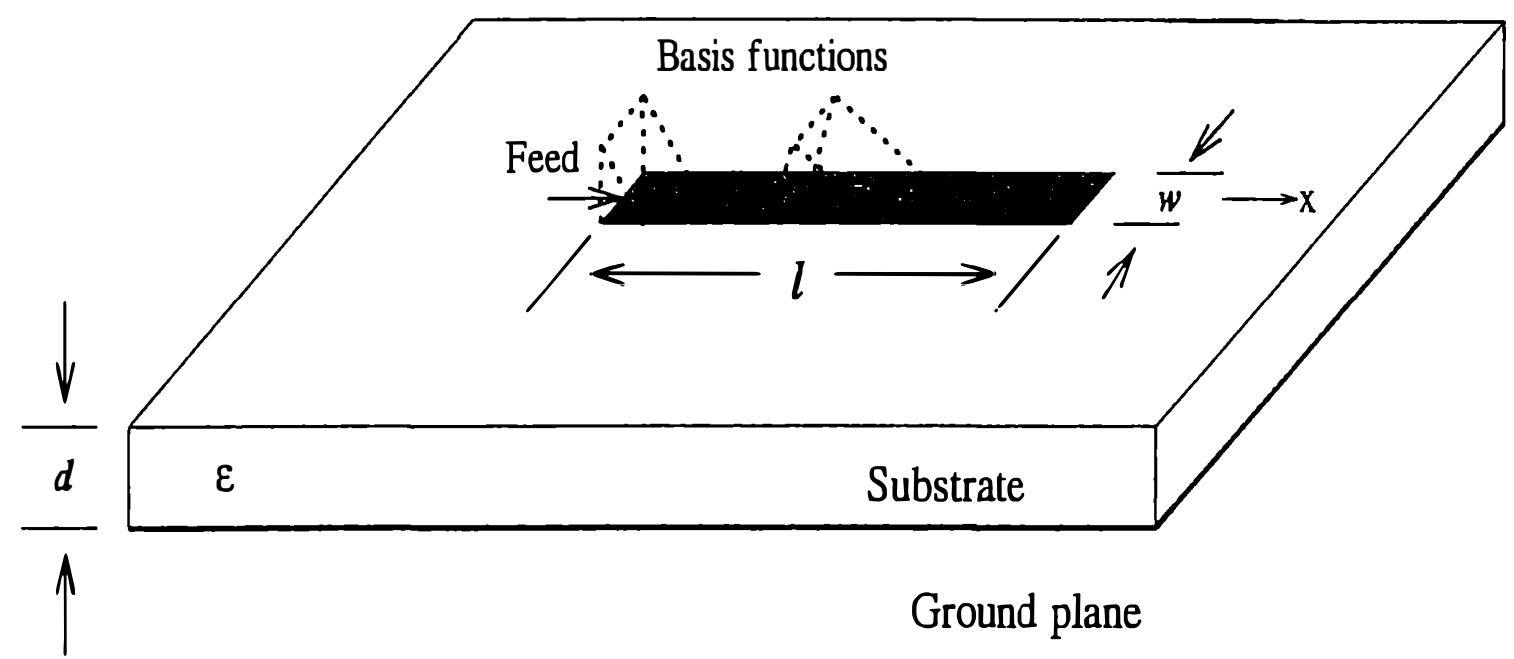

Figure 14: A typical microstrip geometry. $\epsilon_{r}=4.0, \mathrm{~d}=0.02032 \mathrm{~cm}, \mathrm{f}=1.0 \mathrm{GHz}$.

the necessary Green's functions in a closed-form, we consider the following parameters for the geometry of a substrate backed by a ground plane, (see Fig. 14). The dielectric constant $\epsilon_{r}$ of the substrate is 4 ; the thickness of the substrate $\mathrm{d}=0.02032 \mathrm{~cm}$; and, the frequency of operation $\mathrm{f}=1.0 \mathrm{GHz}$. The Green's function of the vector and scalar potentials due to an HED located at the air-substrate interface are obtained in closed forms via the two-level approximation scheme, and the coefficients $a_{1 n}, a_{2 n}$ and the exponents $\alpha_{1 n}, \alpha_{2 n}$ are given in Table 1 along with the normalized Green's functions for vector and scalar potentials, $4 \pi G_{x x}^{A} / \mu_{\mathrm{o}}$ and $4 \pi \epsilon_{\mathrm{o}} G_{x}^{q_{e}}$, respectively, in Fig. 15 . The mixed-potential integral equation for the microstrip line can be transformed into the 
Table 1. Coefficients and Exponents for the closed-form Green's functions

\begin{tabular}{|c|c|c|c|}
\hline \multicolumn{2}{|c||}{$4 \pi G_{x x}^{A} / \mu_{0}=\sum_{n=1}^{N_{1}=4} a_{n} e^{-j k_{s} r_{n}} / r_{n}$} & $4 \pi \epsilon_{o} G_{x}^{q_{e}}=\sum_{n:=1}^{N_{2}=8} a_{n} e^{-j k_{s} r_{n}} / r_{n}$ \\
\hline$a_{n}$ & $\alpha_{n}$ & $a_{n}$ & $\alpha_{n}$ \\
\hline$-1.0000+\mathrm{j} 0.0000$ & $0.000+\mathrm{j} 0.0406$ & $-0.590623+\mathrm{j} 0.0$ & $0.0-\mathrm{j} 0.103 \mathrm{e}-3$ \\
\hline $0.495 \mathrm{e}-6-\mathrm{j} 0.123 \mathrm{e}-5$ & $-0.0512+\mathrm{j} 0.0505$ & $0.0196+\mathrm{j} 0.0374$ & $0.0390+\mathrm{j} 0.0598$ \\
\hline $0.498 \mathrm{e}-6+\mathrm{j} 0.123 \mathrm{e}-5$ & $0.0511+\mathrm{j} 0.0506$ & $-0.4485+\mathrm{j} 0.0$ & $0.0+\mathrm{j} 0.0345$ \\
\hline $1.0+\mathrm{j} 0.0$ & $0.0+\mathrm{j} 0.0$ & $0.0196-\mathrm{j} 0.0374$ & $-0.0390+\mathrm{j} 0.0598$ \\
\hline & & $-0.260 \mathrm{e}-10+\mathrm{j} 0.771 \mathrm{e}-11$ & $-53.33+\mathrm{j} 439.67$ \\
\cline { 2 - 4 } & $0.279 \mathrm{e}-5-\mathrm{j} 0.245 \mathrm{e}-6$ & $-0.3396+\mathrm{j} 0.9239$ \\
\cline { 2 - 4 } & $0.204 \mathrm{e}-6+\mathrm{j} 0.328 \mathrm{e}-6$ & $1.0270-\mathrm{j} 0.2907$ \\
\cline { 2 - 4 } & $1.0+\mathrm{j} 0.0$ & $0.0+\mathrm{j} 0.0$ \\
\hline
\end{tabular}

matrix equation via the spatial-domain MoM, and a typical matrix element is given below to help demonstrate the use of the formulation;

$$
<T_{x}^{\left(m^{\prime}\right)}, G_{x x}^{A} * B_{x}^{(m)}>+\frac{1}{\omega^{2}}<T_{x}^{\left(m^{\prime}\right)}, \frac{\partial}{\partial x}\left(G_{x}^{q_{e}} * \frac{\partial}{\partial x} B_{x}^{(m)}\right)>
$$

The first inner product of (50) is written explicitly as

$$
\begin{aligned}
& <T_{x}^{\left(m^{\prime}\right)}, G_{x x}^{A} * B_{x}^{(m)}>= \\
& \quad \int_{D_{T}} \int d x d y T_{x}^{\left(m^{\prime}\right)}(x, y) \int_{D_{B}} \int d x^{\prime} d y^{\prime} G_{x x}^{A}\left(x-x^{\prime}, y-y^{\prime}\right) B_{x}^{(m)}\left(x^{\prime}, y^{\prime}\right)
\end{aligned}
$$

where $D_{T}$ and $D_{B}$ denote the domains of the testing and basis functions, respectively, and the closed-form Green's function $G_{x x}^{A}$ is expressed as in (39). By changing the order of integration, the inner product can be written

$$
\iint d u d v G_{x x}^{A}(u, v) \iint d x d y T_{x}^{\left(m^{\prime}\right)}(x, y) B_{x}^{(m)}(x-u, y-v)
$$

where the inner double integral is a correlation function represented as $T_{x}^{\left(m^{\prime}\right)} \otimes B_{x}^{(m)}$. As is well-known, the choice of the basis and testing functions are of great importance for the accuracy of the results and for the convergence of the matrix elements involved in the $\mathrm{MoM}^{37}$. Since the formulation presented here requires the correlation function to be polynomial function, the choice of testing and basis functions is restricted to polynomial like functions. Therefore, we choose the rooftop functions, which are triangular functions in the longitudinal direction and uniform in the transverse direction, as the basis and testing functions. Half rooftop functions are also used to model the current density at the load and source terminals ${ }^{38}$.

For the above choice of the basis and testing functions, the correlation function becomes

$$
T_{x}^{\left(m^{\prime}\right)} \otimes B_{x}^{(m)}=f(u) g(v)
$$




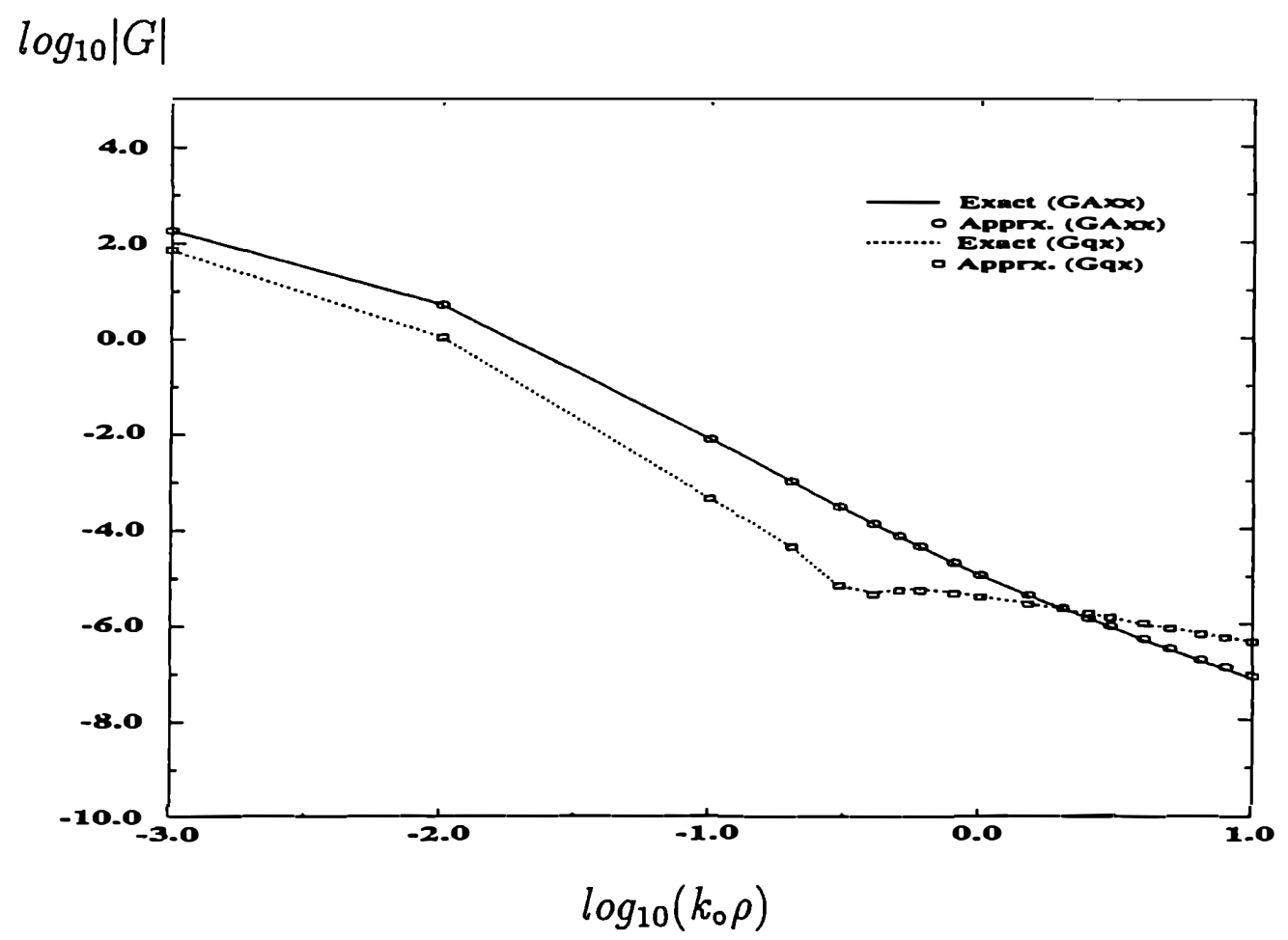

Figure 15: The magnitude of the normalized Green's function $4 \pi G_{x x}^{A} / \mu_{\mathrm{o}}, 4 \pi \epsilon_{\mathrm{o}} G_{x}^{q_{e}}$. $\epsilon_{r}=4.0, \mathrm{~d}=0.02032 \mathrm{~cm}, \mathrm{f}=1.0 \mathrm{GHz}$.

where

$$
\begin{aligned}
& f(u)=\gamma_{3} u^{3}+\gamma_{2} u^{2}+\gamma_{1} u+\gamma_{0} \quad(m-n-2) h_{x}<u<(m-n+2) h_{x} \\
& g(v)=\frac{1}{h_{y}^{2}}\left\{\begin{aligned}
v+h_{y} \quad-h_{y} \leq v \leq 0 \\
-v+h_{y} \quad 0 \leq v \leq h_{y}
\end{aligned}\right.
\end{aligned}
$$

and $\gamma_{0}, \gamma_{1}, \gamma_{2}, \gamma_{3}$ are constants determined by $m, n$ and $h_{x}$ (the half-span of the basis functions in $x$-direction). Also, $h_{y}$, the length of the unit cell in $y$-direction is chosen to equal the width of the microstrip line for the specific geometry considered herein. It should be noted that the formulation given here is also valid for a two-dimensional patch since the order of the polynomials in (54) is the highest that can be encountered in the analysis. By substituting the correlation function (53) and the Green's function (39) into (52), the inner-product term can be written as

$$
\begin{aligned}
\frac{1}{h_{y}^{2}} \sum_{n=1}^{N} a_{n} & {\left[\gamma_{0}\left(h_{y} \iint \frac{e^{-j k_{s} r_{n}}}{r_{n}} d v d u-\iint \frac{e^{-j k_{s} r_{n}}}{r_{n}} v d v d u\right)\right.} \\
+ & \gamma_{1}\left(h_{y} \iint \frac{e^{-j k_{s} r_{n}}}{r_{n}} u d v d u-\iint \frac{e^{-j k_{s} r_{n}}}{r_{n}} u v d v d u\right) \\
+ & \gamma_{2}\left(h_{y} \iint \frac{e^{-j k_{s} r_{n}}}{r_{n}} u^{2} d v d u-\iint \frac{e^{-j k_{s} r_{n}}}{r_{n}} u^{2} v d v d u\right) \\
+ & \left.\gamma_{3}\left(h_{y} \iint \frac{e^{-j k_{s} r_{n}}}{r_{n}} u^{3} d v d u-\iint \frac{e^{-j k_{s} r_{n}}}{r_{n}} u^{3} v d v d u\right)\right]
\end{aligned}
$$


where $r_{n}$ is either $r_{1 n}$ or $r_{2 n}$ as defined in (39).

Because the integrals in Eq. (55) occur in the calculation of matrix elements, and because they cannot be evaluated analytically, their numerical evaluations constitute almost the entire fill time of the MoM matrix. Although the use of the closedform Green's functions in conjunction with the MoM improves the matrix fill time significantly ${ }^{38}$, it could be further improved if the integrals involved (55) can be evaluated analytically. It has been shown that the Taylor's series expansion of the exponential term in the integrand of the first integral in (55) results in an analytically integrable function over a surface ${ }^{39}$. Using this fact, and some of the integral identities ${ }^{40}$, we can evaluate the integrals in (55) analytically. The case of the Taylor's series expansion requires examining its convergence for all $r_{n}$ values with the same number of terms; therefore the expansion is performed around different center points $R_{c}$ for different regions corresponding to different basis and testing function pairs. The $m^{\text {th }}$ order Taylor series expansion of $f(z)$ around $z_{\circ}$ involves an error term of

$$
\text { error }=\left|\frac{f^{m+1}(c)}{(m+1) !}\left(z-z_{o}\right)^{m+1}\right|
$$

where $\mathrm{c}$ is a point in the region of convergence. As the $m^{\text {th }}$ derivative of $e^{-j k_{s} r_{n}}$ is bounded by $k_{s}^{m}$, the error introduced by using the $m^{\text {th }}$ order expansion is

$$
\text { error } \leq \frac{\left|k_{s}\left(r_{n}-R_{c}\right)\right|^{m+1}}{(m+1) !}
$$

It can be observed that the distance to the center point directly determines the error. Hence, to minimize the error, the locations of the center points are chosen to be the mid-point of the each integration region for which the integration intervals are $2 h_{y}$ and $4 h_{x}$ for $\mathrm{v}$ and $\mathrm{u}$ integrations, respectively, and consequently, $r_{n}-R_{c} \leq \sqrt{\left(2 h_{x}\right)^{2}+h_{y}^{2}}$. For the choice of 20 basis functions for each wavelength, $r_{n}-R_{c}$ is bounded by $0.11 \lambda$ and the error is obtained in the following form

$$
\text { error } \leq \frac{(2 \pi \times 0.11)^{m+1}}{(m+1) !}=\frac{(0.7)^{m+1}}{(m+1) !}
$$

from which it is easily shown that an error bounded by $10^{-4}$ can be obtained with the use of at least five terms of the Taylor series. The results presented in Section 3.4 demonstrate that the amount of error of $10^{-4}$ or smaller does not affect the end result as the current distributions obtained by using analytic and numeric integrations are in good agreement. Hence, it is not worthwhile to increase the number of terms any further.

Using the fifth order Taylor series expansion around $R_{c}, e^{-j k_{s} r_{n}}$ can be approximated as

$$
e^{-j k_{s} r_{n}} \approx e^{-j k_{s} R_{c}}\left(\beta_{0}+\beta_{1} r_{n}+\beta_{2} r_{n}^{2}+\beta_{3} r_{n}^{3}+\beta_{4} r_{n}^{4}+\beta_{5} r_{n}^{5}\right)
$$


where

$$
\begin{aligned}
& \beta_{0}=\left(1+j k_{s} R_{c}-\frac{k_{s}^{2} R_{c}^{2}}{2}-j \frac{k_{s}^{3} R_{c}^{3}}{6}+\frac{k_{s}^{4} R_{c}^{4}}{24}+j \frac{k_{s}^{5} R_{c}^{5}}{120}\right) \\
& \beta_{1}=\left(-j k_{s}+k_{s}^{2} R_{c}+j \frac{k_{s}^{3} R_{c}^{2}}{2}-\frac{k_{s}^{4} R_{c}^{3}}{6}-j \frac{k_{s}^{5} R_{c}^{4}}{24}\right) \\
& \beta_{2}=\left(-\frac{k_{s}^{2}}{2}-j \frac{k_{s}^{3} R_{c}}{2}+\frac{k_{s}^{4} R_{c}^{2}}{4}+j \frac{k_{s}^{5} R_{c}^{3}}{12}\right) \\
& \beta_{3}=\left(j \frac{k_{s}^{3}}{6}-\frac{k_{s}^{4} R_{c}}{6}-j \frac{k_{s}^{5} R_{c}^{2}}{12}\right) \\
& \beta_{4}=\left(\frac{k_{s}^{4}}{24}+j \frac{k_{s}^{5} R_{c}}{24}\right) \\
& \beta_{5}=-j \frac{k_{s}^{5}}{120}
\end{aligned}
$$

Replacing the exponential term $e^{-j k_{s} r_{n}}$ in (55) by its Taylor series expansion given in (57) requires an analytic evaluation of the integrals of the type

$$
\iint r_{n}^{j} u^{k} v^{l} d u d v \quad \text { for } \mathrm{j}=-1,0, \ldots, 4, \mathrm{k}=0, \ldots, 3, \mathrm{l}=0,1
$$

which are given in Appendix B. Note that the same procedure presented above can be applied to the second inner-product term of (50) in which $G_{x}^{q_{e}}$ has the same functional form as $G_{x x}^{A}$ given in (39).

\subsection{Results and Discussions}

In this part of the study, the formulation described above is applied to a microstrip line to evaluate computational efficiency. The dielectric constant of the medium is $\epsilon_{r}$ $=4.0$, the ratio of line width $\mathrm{w}$ to substrate thickness $\mathrm{d}$ is 4.0 . The thickness of the substrate is $0.02032 \mathrm{~cm}(=8.0$ mils. $)$, the frequency is $1 \mathrm{GHz}$, and the length of the line is $10 \mathrm{~cm}$. Computational efficiency of the proposed method is assessed in terms of the CPU time obtained on a SUNsparc-10 workstation.

The current distribution on the microstrip line is obtained by numerically integrating the double integrals (52) involved in the MoM matrix elements (Case 1), and then by using the analytic integration formulation presented in Section 3.3 (Case 2). The current distributions derived via the use of the numerical integration (Case 1) have been verified by comparing it with the results obtained via the transmission line method $^{38}$; therefore, Case 1 serves here as a reference for the accuracy of the current distribution as well. In the numerical integration, 16-point Gauss quadrature integration algorithm, which is considered to be one of the fastest numerical integration algorithms, is employed for the double integrals for which the range of the integration is divided into sub-regions to guarantee the convergence of the numerical integration. For both cases, the CPU times are obtained for different numbers of basis functions 


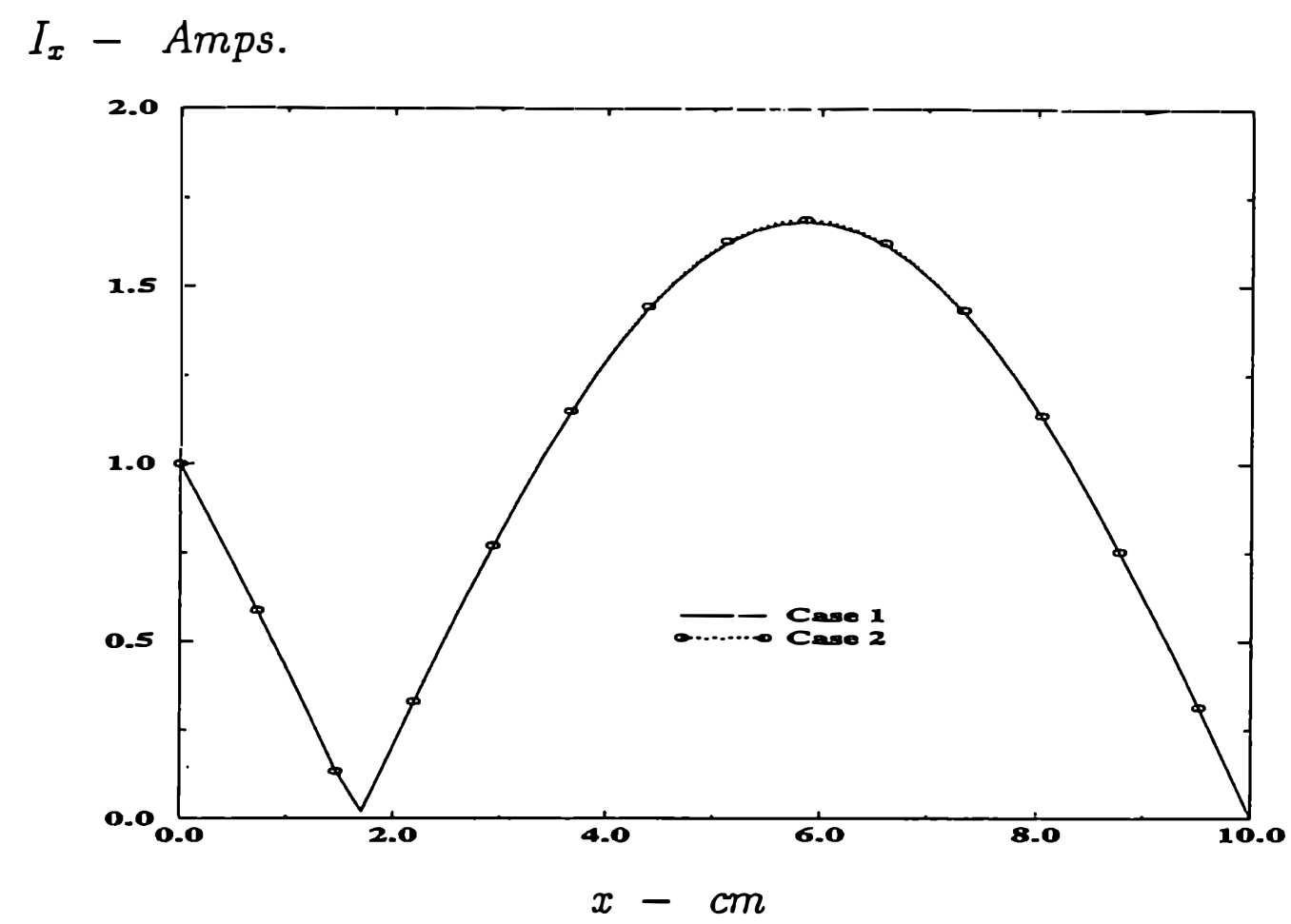

Figure 16: Current distributions obtained by using 40 basis functions. $\epsilon_{r}=4.0$, $\mathrm{d}=0.02032 \mathrm{~cm}, \mathrm{f}=1.0 \mathrm{GHz}, \mathrm{w}=0.08128 \mathrm{~cm}, \mathrm{l}=10 \mathrm{~cm}$.

and are listed in Table 2. The current distributions obtained via numerical and analytical evaluations of the integrals for 40 basis functions are shown in Fig. 16. It

Table 2. CPU times for different number of basis functions

\begin{tabular}{|c|c|c|}
\hline number of basis func. & CPU time in sec. (Case 1) & CPU time in sec. (Case 2) \\
\hline 10 & 13.3 & 0.36 \\
\hline 20 & 27.0 & 0.65 \\
\hline 30 & 39.0 & 0.98 \\
\hline 40 & 52.95 & 1.33 \\
\hline
\end{tabular}

can be observed from Table 2 that the elimination of the numerical integrals reduces the computation time approximately by a factor of 40 . Besides the improvement in computational efficiency, the formulation based on the analytical integration also provides a number of other advantages. First of all, as the MoM becomes a technique free from any numerical integrations; hence, the numerical errors due to integration as well as the effort needed to locate an appropriate numerical integration algorithm are eliminated. Secondly, as the matrix entries are expressed in closed-forms, the effect of changing the geometrical parameters, e.g., the length and width of the microstrip line, on the output parameters such as current distribution, input impedance or spurious radiation, can be studied analytically by taking a derivative with respect to the desired parameter. Finally, if a method uses numerical integrations, it is nec- 
essary to extract the singularity at the source point, while in the analytic integration formulation this problem is completely eliminated because the singularities involved are integrable over a surface. It should be noted that the application of the proposed approach here has no restriction for the size of the geometry, provided the closed-form Green's functions are valid for the distance as far as the maximum distance of the geometry.

\section{Conclusions}

The closed-form Green's functions, developed in the past, suffer from the drawback of choosing the parameters for the exponential approximation procedure required in its derivation. Moreover, the extraction of the SWPs and real images may not be possible or efficient for multilayer geometries when the original version of the approach is employed. Here, a new approach based on a two-level approximation is proposed to overcome these difficulties and to make the use of closed-form Green's functions attractive for those developing the EM software as well as for researchers in the field. The major advantages of this approach are its robustness and the computational efficiency, both of which are demonstrated in the text. Numerical examples of the closed-form Green's functions are given for a multilayer medium. The approximate Green's functions are compared with the exact ones and very good agreement is achieved.

When the spatial domain MoM is used in conjunction with the closed-form Green's functions for the solution of the mixed-potential integral equation, the MoM matrix elements involve two-dimensional integrals whose numerical evaluations increase the matrix fill-time. In order to improve the numerical efficiency of the method, the integrand is approximated by its Taylor series and each term of the expansion is integrated analytically. By eliminating the numerical integration from the MoM, the matrix fill-time is decreased drastically, which is a significant improvement in the matrix-fill time of the MoM. This acceleration in the matrix fill time makes the MoM an efficient and full-wave analysis technique, which can be utilized in an optimization algorithm for the solution of a microstrip circuit or antenna design problem. The proposed method offers other advantages as well. For instance, by expressing the matrix entries in closed-forms, it becomes possible to investigate the effect of varying certain parameters on the end result by examining the matrix entries and their derivatives analytically.

\section{Appendix A: Alternative Form of Green's Function}

Since the vector and scalar potentials are not uniquely defined in stratified media, different sets of Green's functions for the vector and scalar potentials are possible, and they lead to several different MPIE formulations ${ }^{31,32}$. Among these Green's functions, three useful choices, referred to as formulations $\mathrm{A}, \mathrm{B}$, and $\mathrm{C}$ were given by 
Michalski ${ }^{33}$. In this paper, the formulation $\mathrm{C}$ is chosen as the alternative form of the Green's function and given here as,

$$
\begin{aligned}
\overrightarrow{\bar{G}}^{A, F} & =(\hat{x} \hat{x}+\hat{y} \hat{y}) G_{x x}+\hat{z} \hat{x} G_{z x}+\hat{z} \hat{y} G_{z y} \\
& +\hat{x} \hat{z} G_{x z}+\hat{y} \hat{z} G_{y z}+\hat{z} \hat{z} G_{z z}
\end{aligned}
$$

and $G^{q_{e, m}}$ as the Green's function for the scalar potential for both horizontal and vertical dipoles, Fig. 1. Note that the difficulties encountered in the traditional formulation due to the difference between the scalar potentials of HED (HMD) and VED (VMD) are alleviated in this formulation. In the above form of the Green's function, the terms associated with the horizontal dipoles $\left(G_{x x}^{A, F}, G_{y y}^{A, F} G_{z x}^{A, F}, G_{z y}^{A, F}\right.$, $G_{x}^{q_{e, m}}$, and $G_{y}^{q_{e, m}}$ ) remain the same as in the traditional form (6)-(11); two new entries, $G_{x z}^{A, F}$ and $G_{y z}^{A, F}$, are introduced, and $G_{z z}^{A, F}$ is modified for the vertical dipoles. For an $x$-oriented HED and a $z$-oriented VED, the Green's function components used with the alternative form, are adopted to the formulation given in Section 2.1 as,

$$
\begin{aligned}
\tilde{G}_{z z}^{A} & =\frac{1}{j k_{z_{i}}}\left[\frac{\mu_{i}}{2} e^{-j k_{z_{i}}|z|}+e^{-j k_{z_{i}} z}\left[\frac{\mu_{i}}{2} A_{v}^{e}-\frac{k_{z_{i}}^{2}}{2 \omega^{2} \epsilon_{i}}\left[A_{v}^{e}\right.\right.\right. \\
& \left.\left.-\frac{k_{i}^{2} C_{h}^{e}-k_{z_{i}}^{2} D_{h}^{e}}{k_{\rho}^{2}}\right]\right]+e^{j k_{z_{i}} z}\left[\frac{\mu_{i}}{2} B_{v}^{e}+\frac{k_{z_{i}}^{2}}{2 \omega^{2} \epsilon_{i}}\left[-B_{v}^{e}\right.\right. \\
& \left.\left.\left.+\frac{k_{z_{i}}^{2} B_{h}^{e}+k_{i}^{2} A_{h}^{e}}{k_{\rho}^{2}}\right]\right]\right] \\
\tilde{G}_{x z}^{A} & =\frac{-j}{k_{z_{i}}} \frac{-k_{x} k_{z_{i}}}{2 \omega^{2} \epsilon_{i}}\left[e^{-j k_{z_{i}} z}\left[A_{v}^{e}-\frac{k_{i}^{2} C_{h}^{e}-k_{z_{i}}^{2} D_{h}^{e}}{k_{\rho}^{2}}\right]\right. \\
& \left.+e^{j k_{z_{i}}}\left[-B_{v}^{e}+\frac{k_{z_{i}}^{2} B_{h}^{e}+k_{i}^{2} A_{h}^{e}}{k_{\rho}^{2}}\right]\right]
\end{aligned}
$$

where, $\tilde{G}_{z z}^{A}, \tilde{G}_{x z}^{A}$ are the alternative Green's function components in the source layer, and $A_{h, v}^{e}, B_{h, v}^{e}, C_{h}^{e}, D_{h}^{e}$ are given in the Eqs. (16)-(21).

6. Appendix B: The Analytic Evaluation of the Integrals Given in (64)

$$
R=\sqrt{u^{2}+v^{2}+c^{2}}
$$

where $\mathrm{c}$ is any of the complex exponents. Some special integrals are defined in order to simplify the formulation.

$$
J_{0}=\int R d u=\frac{1}{2}\left(R u+\left(v^{2}+c^{2}\right) \log (u+R)\right)
$$




$$
\begin{aligned}
& J_{1}=\int u R d u=\frac{R^{3}}{3} \\
& J_{2}=\int u^{2} R d u=\frac{u\left(v^{2}+c^{2}+2 u^{2}\right) R}{8}-\frac{\left(v^{2}+c^{2}\right)^{2}}{8} \log (u+R) \\
& J_{3}=\int u^{3} R d u=\frac{R^{5}}{5}-\left(v^{2}+c^{2}\right) \frac{R^{3}}{3} \\
& J_{4}=\int u^{4} R d u=\left(-\frac{3}{2}\left(v^{2}+c^{2}\right)^{2}+\left(v^{2}+c^{2}\right) u^{2}+4 u^{4}\right)\left(\frac{u R}{24}\right) \\
& +\frac{1}{16}\left(v^{2}+c^{2}\right)^{3} \log (u+R) \\
& J_{5}=\int u^{5} R d u=\frac{R^{7}}{7}-\frac{2}{5} R^{5}\left(v^{2}+c^{2}\right)+\frac{R^{3}}{3}\left(v^{2}+c^{2}\right)^{2} \\
& J_{6}=\int u^{6} R d u=\frac{7 u}{8} J_{5}-\frac{\left(v^{2}+c^{2}\right)}{40} J_{4}+\frac{\left(v^{2}+c^{2}\right)^{2}}{30} J_{2}-\frac{\left(v^{2}+c^{2}\right)^{3}}{15} J_{0} \\
& J_{7}=\int u^{7} R d u=\frac{R^{9}}{9}-3\left(v^{2}+c^{2}\right) \frac{R^{7}}{7}+3\left(v^{2}+c^{2}\right)^{2} \frac{R^{5}}{5}-\left(v^{2}+c^{2}\right)^{3} \frac{R^{3}}{3} \\
& K_{0}=\int \frac{1}{R} d u=\log (u+R) \\
& K_{1}=\int \frac{u}{R} d u=R \\
& K_{n}=\int \frac{u^{n}}{R} d u=J_{n-2}-\left(v^{2}+c^{2}\right) K_{n-2} \\
& I_{0}=\int \frac{1}{\left(u^{2}+c^{2}\right) R} d u=\frac{1}{\underline{v c}} \arctan \left(\frac{u v}{c R}\right) \\
& I_{1}=\int \frac{u}{\left(u^{2}+c^{2}\right) R} d u=\frac{\log (R-v)-\log (R+v)}{2 v} \\
& I_{n}=\int \frac{u^{n}}{\left(u^{2}+c^{2}\right) R} d u=K_{n-2}-c^{2} I_{n-2} \\
& L_{0}=\int \frac{1}{\left(u^{2}+c^{2}\right)} d u=\frac{1}{c} \arctan \left(\frac{u}{c}\right) \\
& L_{1}=\int \frac{u}{\left(u^{2}+c^{2}\right)} d u=\frac{\log \left(u^{2}+c^{2}\right)}{2} \\
& L_{n}=\int \frac{u^{n}}{\left(u^{2}+c^{2}\right)} d u=\frac{u^{n-1}}{n-1}-c^{2} L_{n-2} \\
& M_{n}=\iint \frac{u^{n}}{R} d v d u=\left(u^{n+1} \log (v+R)-L_{n+2}+v I_{n+2}\right) /(n+1) \\
& \iint u^{n} R d v d u=\frac{1}{2}\left(v J_{n}+M_{n+2}+c^{2} M_{n}\right) \\
& \iint u^{n} R^{2} d v d u=\frac{u^{n+1}}{n+1}\left(\frac{v^{3}}{3}+v c^{2}\right)+\frac{u^{n+3}}{n+3} v
\end{aligned}
$$




$$
\begin{aligned}
\iint u^{n} R^{3} d v d u & =\frac{5}{8} v J_{n+2}+\frac{v}{4}\left(\frac{5 c^{2}}{2}+v^{2}\right) J_{n}+\frac{3}{8}\left(M_{n+4}+2 c^{2} M_{n+2}+c^{4} M_{n}\right) \\
\iint u^{n} R^{4} d v d u & =\frac{u^{n+1}}{n+1}\left(c^{4} v+\frac{v^{5}}{5}+\frac{2 c^{2} v^{3}}{3}\right)+\frac{2 u^{n+3}}{n+3}\left(\frac{v^{3}}{3}+c^{2} v\right)+\frac{v u^{n+5}}{n+5} \\
\iint \frac{u^{n} v}{R} d v d u & =J_{n} \\
\iint u^{n} v R d v d u & =\frac{J_{n+2}}{3}+\frac{\left(v^{2}+c^{2}\right) J_{n}}{3} \\
\iint u^{n} v R^{2} d v d u & =\frac{u^{n+3} v^{2}}{2(n+3)}+\frac{v^{2}}{2}\left(c^{2}+\frac{v^{2}}{2}\right) \frac{u^{n+1}}{n+1} \\
\iint u^{n} v R^{3} d v d u & =\frac{J_{n+4}}{5}+\frac{2\left(v^{2}+c^{2}\right)}{5} J_{n+2}+\frac{\left(v^{2}+c^{2}\right)^{2}}{5} J_{n} \\
\iint u^{n} v R^{4} d v d u & =\frac{u^{n+5} v^{2}}{2(n+5)}+\frac{u^{n+3}}{n+3}\left(c^{2} v^{2}+\frac{v^{4}}{2}\right)+\frac{u^{n+1}}{n+1}\left(\frac{c^{4} v^{2}}{2}+\frac{v^{6}}{6}+\frac{c^{2} v^{4}}{2}\right)
\end{aligned}
$$

\section{Acknowledgments}

The authors would like to acknowledge the contributions made by Dr. G. Dural and L. Alatan who have previously worked on this project or with whom we have consulted over the course of the preparation of this paper.

\section{References}

1. N. K. Das and D. M. Pozar, IEEE Trans. Microwave Theory Tech. MTT-35 (1987) 326.

2. F. Crog and D. M. Pozar, IEEE Trans. Antennas Propagat. AP-40 (1992) 1367.

3. L. Barlatey, J.R. Mosig and T. Sphicopoulos, IEEE Trans. Antennas Propagat. AP-18 (1990) 608 .

4. A. N. Tulintseff, S. M. Ali and J. A. Kong, IEEE Trans. Antennas Propagat. AP-39 (1991) 381 .

5. K. R. Carver and J. W. Mink, IEEE Trans. Antennas Propagat. AP-29 (1981) 2.

6. D. R. Jackson, N. G. Alexopoulos, IEEE Trans. Antennas Propagat. AP-33 (1985) 976.

7. C. I. G. Hsu, R. F. Harrington, K. A. Michalski, D. Zheng, it IEEE Trans. Microwave Theory Tech. MTT-41 (1993) 70. 
8. M. Gillick, I. D. Robertson, J. S. Joshi, it IEEE Trans. Microwave Theory Tech. MTT41 (1993) 346.

9. R. F. Harrington, Field Computation by Moment Methods. (Krieger Publishing, New York, 1983).

10. J. Jin, The Finite Element Method in Electromagnetics. (John Willey \& Sons, Inc, New York, 1993).

11. K. Kunz and R. Luebber, The Finite Difference Time Domain Method for Electromagnetics. (CRC Press, Boca Raton, FL, 1993).

12. R. Schinzinger and P. Laura, Conformal Mapping: Methods and Applications. (Elsevier, New York, 1991).

13. A. Sommerfeld, Partial Differential Equations in Physics. (Academic Press Inc., New York, 1949).

14. T. Itoh and R. Mittra, IEEE Trans. Microwave Theory Tech. MTT-21 (1973) 496.

15. M. D. Deshpande and M. C. Bailey, IEEE Trans. Antennas and Propagat. AP-30 (1982) 645.

16. D. M. Pozar, IEEE Trans. Antennas and Propagat. AP-30 (1982) 1191.

17. D. R. Jackson and N. G. Alexopoulos, IEEE Trans. Antennas and Propagat. AP-34 (1986) 1430.

18. S. M. Wright, Efficiency and Analysis of Infinite Microstrip Arrays on Electrically Thich Substrate (Ph. D. dissertation, University of Mlinois at Urbana-Champaign, 1984).

19. M. I. Aksun, S. L. Chuang and Y. T. Lo, J. Electromagn. Waves Appl. 6 (1992) 341.

20. J. R. Mosig and F. E. Gardiol, Advances in Electronics and Electron Physics 59 (1982) 139 .

21. D. C. Chang, D. I. Wu and J. X. Zheng, in Directions in Electromagnetic Wave Modeling, eds. H. L. Bertoni and L. B. Felsen (Plenum, New York, 1991).

22. Y. L. Chow, J. J. Yang, and D. F. Fang and G. E. Howard, IEEE Trans. Microwave Theory Tech. MTT-39 (1991) 588.

23. M. I. Aksun and R. Mittra, IEEE Trans. Microwave Theory Tech. MTT-40 (1992) 2055.

24. G. Dural and M. I. Aksun, IEEE Trans. Microwave Theory Tech. in Press.

25. M. I. Aksun, IEEE Trans. Microwave Theory Tech. in Press.

26. S. L. Marple, Digital Spectral Analysis with Applications ( Prentice Hall, New Jersey, 1987). 
27. A. J. Mackay, A. McCowen, IEEE Trans. Antennas and Propagat. AP-35 (1987) 435.

28. Y. Hua and T. K. Sarkar, IEEE Trans. Antennas Propagat. AP-37 (1989) 229.

29. M. I. Aksun and R. Mittra, IEEE Trans. Electromagn. Compat. EMC-35 (1993) 148.

30. L. Alatan, M. I. Aksun, K. Mahadevan and T. Birand, IEEE Trans. Microwave Theory Tech. in Press.

31. A. Erteza and. B. K. Park, it IEEE Trans. Antennas Propagat. AP-17 (1969) 376.

32. K. A. Michalski, IEEE Trans. Antennas Propagat. AP-35 (1987) 1299.

33. K.A.Michalski, D. Zheng, IEEE Trans. Antennas Propagat. AP-38 (1990) 335.

34. W. C. Chew, Waves and Fields in Inhomogeneous Media (Van Nostrad Reinhold, New York, 1990).

35. S. Barkeshli, P. H. Pathak and M. Marin, IEEE Trans. Antennas Propagat. AP-38 (1990) 1374.

36. L. B. Felsen and N. Marcuvitz, Radiation and Scattering of Waves (Prentice-Hall, New Jersey, 1973.)

37. M. I. Aksun and R. Mittra, IEEE Trans. Microwave Theory Tech. MTT-41 (1993) 503.

38. M. I. Aksun and R. Mittra, IEEE Trans. Microwave Theory Tech. MTT-41 (1992) 148.

39. R. F. Harrington, Proc. IEEE 55 (1967) 139.

40. K. Mahadevan and H. A. Auda, IEEE Trans. Antennas Propagat. AP-37 (1989) 1503. 